\title{
THE BROWNIAN MOTION AND THE CANONICAL STOCHASTIC FLOW ON A SYMMETRIC SPACE
}

\author{
MING LIAO
}

\begin{abstract}
We study the limiting behavior of Brownian motion $x_{t}$ on a symmetric space $V=G / K$ of noncompact type and the asymptotic stability of the canonical stochastic flow $F_{t}$ on $O(V)$. We show that almost surely, $x_{t}$ has a limiting direction as it goes to infinity. The study of the asymptotic stability of $F_{t}$ is reduced to the study of the limiting behavior of the adjoint action on the Lie algebra $\mathscr{G}$ of $G$ by the horizontal diffusion in $G$. We determine the Lyapunov exponents and the associated filtration of $F_{t}$ in terms of root space structure of $\mathscr{G}$.
\end{abstract}

\section{INTRODUCTION}

The purpose of this paper is to study the asymptotic behavior of the Brownian motion and the canonical stochastic flow on a Riemannian symmetric space $V$. If $V$ is of compact type, then the Brownian motion is known to be recurrent and we will show that the canonical stochastic flow has only zero Lyapunov exponent. The case of noncompact type symmetric spaces is more interesting.

It is well known that the Brownian motion on a simply connected Riemannian manifold whose sectional curvatures are bounded from above and below by negative numbers has an asymptotic direction, see [9 and 10]. This is not true if some of the sectional curvatures are allowed to be zero. In fact, the proof of this result cannot be applied to a Riemannian symmetric space of noncompact type, whose sectional curvatures are only nonpositive and some of them may be zero. However, in this case, the Brownian motion has an asymptotic direction. This is a direct consequence of the limiting behavior of the horizontal diffusion process associated with a symmetric space with respect to the Cartan decomposition, see [6]. That paper also discusses the limiting behavior with respect to the Iwasawa decomposition. In fact, the result for the Cartan decomposition is derived from that for the Iwasawa decomposition. See also [11 and 12] for a more accessible treatment of these results. In the present paper, we will present a different and more direct proof for the existence of the asymptotic direction of Brownian motion on a noncompact type symmetric space.

Our main purpose is to determine the Lyapunov exponents and the associated

Received by the editors September 10, 1990 and, in revised form, October 14, 1991.

1980 Mathematics Subject Classification (1985 Revision). Primary 58G32, 60J65.

The author was on leave of absence from Nankai University, Tianjin, PR China.

Research supported by Hou Ying Dong Educational Foundation of PRC and an NSF summer research grant. 
filtration for the canonical stochastic flow on the orthonormal frame bundle of a symmetric space of noncompact type.

We will now briefly recall some basic definitions. Let $V$ be a $d$-dimensional Riemannian manifold and let $O(V)$ be its orthonormal frame bundle. For any orthonormal frame $u=\left(X_{1}, X_{2}, \ldots, X_{d}\right)$ at $o \in V$, let $u(t)$ be the parallel displacement of $u$ along a curve tangent to $X_{j}$ at $o . u(t)$ is a curve in $O(V)$. Let $\bar{X}_{j}(u)$ be the tangent of $u(t)$ at $u$. Then $\bar{X}_{1}, \bar{X}_{2}, \ldots, \bar{X}_{d}$ are $n$ vector fields on $O(V)$. The stochastic flow $F_{t}$ generated by the Stratonovich stochastic differential equation

$$
d u_{t}=\sum_{j=1}^{d} \bar{X}_{j}\left(u_{t}\right) \circ d w_{t}^{j}
$$

is called the canonical stochastic flow on $O(M)$, where $w_{t}=\left(w_{t}^{1}, w_{t}^{2}, \ldots, w_{t}^{d}\right)$ is a $d$-dimensional Wiener process. Let $u$ be an orthonormal frame at $o$. The image of $F_{t}(u)$ under the natural projection: $O(V) \rightarrow V$ is a Brownian motion in $V$, i.e., a diffusion process with one half Laplacian as generator.

Let $\|\cdot\|_{c}$ be the canonical metric on $O(V)$ defined in [2]. The canonical metric "respects" the Riemannian connection. If $g: V \rightarrow V$ is an isometry, then it induces naturally a map $g: O(V) \rightarrow O(V)$, which is isometric with respect to $\|\cdot\|_{c}$. The Lyapunov exponents of $F_{t}$ are defined as the limits of $(1 / t) \log \left\|D F_{t}(X)\right\|_{c}$ as $t \rightarrow \infty$, where $D F_{t}$ is the differential map of $F_{t}$ and $X \in T O(V)$.

The Lyapunov exponents describe the asymptotic stability of the canonical stochastic flow and are interesting geometric invariants. A general discussion can be found in [2]. In the case of constant curvature manifolds, the Lyapunov exponents are computed explicitly in [1] and [2]. Note that in the present case, the existence of exponents is not guaranteed by the general theory since the manifold is not compact.

We will take an algebraic approach as in Baxendale [1]. But unlike [1] which relies heavily on explicit computations, we will use the general theory of symmetric spaces. As in the case of constant curvature, the computation of exponents of the canonical stochastic flow can be reduced to computing the exponents of the adjoint action of the horizontal diffusion. One will need the existence of the asymptotic direction of Brownian motion and the asymptotic growth rate of the radial part of Brownian motion. In fact, the filtration associated with the exponents depends on the asymptotic direction of Brownian motion and this dependence can be described in terms of the root space structure of the Lie algebra. At one crucial step, one will have to carefully examine the exponential rate at which the Brownian motion converges to its asymptotic direction and the effect on the adjoint action of the horizontal diffusion. This is now more difficult than in the case of constant curvature because the root system of the Lie algebra is more complicated.

Our paper generalizes the results in the first part of [1] for hyperbolic spaces to general symmetric spaces. In the second part of [1], the stability of the gradient flow on the sphere $S^{d-1}$ is discussed using the fact that the gradient flow can be induced by the horizontal diffusion in $S O(d, 1)$. This second part can also be generalized and the gradient flow on $S^{d-1}$ corresponds to the stochastic flows on the boundaries of a Lie group $G$ induced by the horizontal diffusion in $G$ (see the author's recent paper [5]). 
To make this paper accessible to a more general audience, we will use the space $V=S L(n) / S O(n)$ to explain the theory of symmetric spaces. This method was used in [11 and 12], see also [7]. The reader may choose to limit his attention only to this example, then a solid knowledge in symmetric spaces is not really necessary.

It should be mentioned that our method only yields the exponents for the flow $F_{t}$ restricted to a subbundle $G_{u}$ of $O(V)$, the orbit of a frame $u$ under the action of $G$, which is invariant under $F_{t} . G_{u}=O(V)$ if $V$ has constant curvature. It seems that $F_{t}$ on $O(V)$ should have the same exponents. At least, this is true if all the exponents of $F_{t}$ on $O(V)$ exist, such is the case if $V$ is compact, see the proof of Theorem 1. But we are unable to prove this in general.

The paper is organized as follows. The next section contains a brief introduction to symmetric spaces. We show that the discussion of the canonical stochastic flow $F_{t}$ can be reduced to that of the horizontal diffusion $g_{t}$ in $G$. Section 3 summarizes some basic facts about symmetric spaces of noncompact type. In $\S 4$, we show that, almost surely, Brownian motion in a noncompact type symmetric space has an asymptotic direction and its radial part has a limiting growth rate. Section 5 contains our main result. We determine the limiting behavior of the adjoint action of the horizontal diffusion (Theorem 3 ) and as a consequence, we obtain the Lyapunov exponents and the associated filtration of the canonical stochastic flow. Section 6 contains the proof of a technical result which is used early.

\section{SYMMETRIC SPACES}

We will briefly recall some facts about symmetric spaces. The reader is referred to [3] for details.

Let $G$ be a connected Lie group with Lie algebra $\mathscr{G}$. Any $X \in \mathscr{G}$ is identified with a left invariant vector field on $G$. For $X, Y \in \mathscr{G}$, their Lie bracket $[X, Y]$ is the element of $\mathscr{G}$ which is identified with the left invariant vector field $X Y-Y X$. We define $\operatorname{ad}(X): \mathscr{G} \rightarrow \mathscr{G}$ by $\operatorname{ad}(X) Y=[X, Y]$. For any $g \in G$, the Lie group automorphism: $G \rightarrow G$ defined by $\psi \mapsto g \psi g^{-1}$ induces a Lie algebra automorphism $\operatorname{Ad}(g): \mathscr{G} \rightarrow \mathscr{G}$. This is called the adjoint action of $G$ on $\mathscr{G}$. We have

$$
\forall X \in \mathscr{G}, \quad e^{\operatorname{ad}(X)}=\operatorname{Ad}\left(e^{X}\right) .
$$

Let $K$ be a compact Lie subgroup of $G$ with Lie subalgebra $\mathscr{K}$ and let $\theta$ be an automorphism on $G$ such that $\theta^{2}$ is the identity map and $I\left(G_{\theta}\right) \subset K \subset G_{\theta}$, where $G_{\theta}$ is the subgroup of $G$ fixed by $\theta$ and $I\left(G_{\theta}\right)$ is the identity component of $G_{\theta}$. The homogeneous space $V=G / K$ is called a (globally) symmetric space. We will use $o$ to denote the coset $K$. Note that $G$ acts naturally on $V: g \in G$ maps $\psi K$ into $g \psi K$. The subset $N$ of $G$ which fixes every point of $V$ is a normal subgroup of $G$ contained in $K$. For simplicity, we will assume that $N=\{e\}$, where $e$ is the identity element of $G$. Then $G$ acts effectively on $V$ in the sense that if $g(x)=x$ for all $x \in V$, then $g=e$. A Riemannian metric on $V$ is said to be $G$-invariant if it is left invariant by the action of $G$. All the $G$-invariant Riemannian metrics have the same Riemannian connection (Corollary 4.3 of [3, Chapter IV]). 
Let $\theta$ also denote the induced Lie algebra automorphism $\theta: \mathscr{G} \rightarrow \mathscr{G}$. For $X \in \mathscr{K}, \theta(X)=X$. Let $\mathscr{P}=\{X \in \mathscr{G} ; \theta(X)=-X\}$. Then $\mathscr{G}=\mathscr{K} \oplus \mathscr{P}$ and $\mathscr{P}$ is $\operatorname{Ad}(K)$-invariant in the sense that $\forall k \in K, \operatorname{Ad}(k) \mathscr{P} \subset \mathscr{P}$. We have

$$
[\mathscr{K}, \mathscr{K}] \subset \mathscr{K}, \quad[\mathscr{K}, \mathscr{P}] \subset \mathscr{P}, \quad[\mathscr{P}, \mathscr{P}] \subset \mathscr{K} .
$$

Note that $\mathscr{P}$ can be identified with $T_{o} V$ under the map $\left.Y \mapsto \frac{d}{d s} \exp (s Y) o\right|_{s=0}$, for $Y \in \mathscr{P}$. The Killing form of $\mathscr{G}$ is defined $B(X, Y)=\operatorname{Trace}(\operatorname{ad}(X) \operatorname{ad}(Y))$ for $X, Y \in \mathscr{G}$. We have $B(X, Y)=0$ for $X \in \mathscr{K}$ and $Y \in \mathscr{P}$, and $B(\cdot, \cdot)$ is negative definite on $\mathscr{K} \times \mathscr{K}$. The homogeneous space $V=G / K$ is said to be a symmetric space of compact type if $(\cdot, \cdot)$ is negative definite on $\mathscr{P} \times \mathscr{P}$, it is said to be of noncompact type if $B(\cdot, \cdot)$ is positive definite on $\mathscr{P} \times \mathscr{P}$.

Let $V=G / K$ be a $d$-dimensional symmetric space of compact or noncompact type. We now introduce an inner product $\langle\cdot, \cdot\rangle$ on $\mathscr{G}$. If $G / K$ is of compact type, we will let $\langle X, Y\rangle=-B(X, Y)$. If $G / K$ is of noncompact type, we will let $\langle X, Y\rangle=-B(X, \theta(Y))$. Since the Killing form $B$ is $\operatorname{Ad}(G)$ invariant, $\langle\cdot, \cdot\rangle$ is $\operatorname{Ad}(G)$-invariant when $G / K$ is of compact type. One can also show easily that $\langle\cdot, \cdot\rangle$ is $\operatorname{Ad}(K)$-invariant when $G / K$ is of noncompact type. We will use $\|X\|$ to denote the square root of $\langle X, X\rangle$. With the identification of $\mathscr{P}$ with $T_{o} V$, the norm $\|\cdot\|$ restricted to $\mathscr{P}$ extends to be a $G$-invariant Riemannian metric on $V$. This Riemannian metric will be called the standard metric on $V$.

Let $G=S L(n)$, the group of $n \times n$ real matrices of determinant one. Note that this is usually denoted by $S L(n, R)$. We will use $S L(n)$ instead to simplify our notation. Let $\theta: G \rightarrow G$ be defined by $\theta(A)=\left(A^{*}\right)^{-1}$, where $A^{*}$ is the transpose of the matrix $A$. The group $G_{\theta}$ coincides with $S O(n)$, the group of $n \times n$ orthogonal matrices of determinant one. We will let $K=S O(n)$. Now $\mathscr{G}=\operatorname{sl}(n)$, the space of $n \times n$ matrices of trace zero, $\mathscr{K}=o(n)$, the space of skew symmetric matrices, and $\mathscr{P}$ is the space of $n \times n$ symmetric matrices of trace zero. Note that the Lie bracket $[X, Y]$ of $X$ and $Y$ is $X Y-Y X$ as matrices and the Lie algebra automorphism $\theta: s l(n) \rightarrow s l(n)$ is given by $\theta(X)=-X^{*}$. The Killing form on $\mathscr{G}=s l(n)$ is given by

$$
B(X, Y)=2 n \operatorname{Trace}(X Y) \text {. }
$$

It follows that $V=S L(n) / S O(n)$ is a symmetric space of noncompact type. We also note that $\langle X, Y\rangle=2 n \operatorname{Trace}\left(X Y^{*}\right)$.

This example will be used throughout to explain our theory. It is much easier to understand the structure theory of symmetric spaces with this example than with a general symmetric space. The reader may choose to work only with this example. Note that $V$ can be identified with the space of positive definite symmetric matrices of determinant one via the map $X \mapsto X X^{*}$ defined on $S L(n)$. Since such a symmetric matrix represents an $n$-dimensional ellipsoid of unit volume, $V$ can be regarded as the space of ellipsoids of unit volume.

Any $g \in G$ is an isometry: $V \rightarrow V$. Its differential map $D g$ induces a map: $O(M) \rightarrow O(M)$, which is isometric with respect to the canonical metric on $O(M)$ and will also be denote by $g$. Let $u$ be an orthonormal frame $\left(X_{1}, \ldots, X_{d}\right)$ at $o$ and let

$$
G_{u}=\{g(u) ; g \in G\} .
$$

$G_{u}$ is a subbundle of $O(V)$. Note that via the map: $G \rightarrow G_{u}$ defined by $g \mapsto g(u), G$ is naturally identified with $G_{u}$ and $\mathscr{G}$ is naturally identified with 
$T_{u} G_{u}$. The canonical metric $\|\cdot\|_{c}$ restricted to $T_{u} G_{u}$ becomes a norm on $\mathscr{G}$, which will still be denoted by $\|\cdot\|_{c}$.

For $g \in G$, the left and right translations $L_{g}$ and $R_{g}: G \rightarrow G$ are defined respectively by $L_{g}(\psi)=g \psi$ and $R_{g}(\psi)=\psi g$. Recall that $X_{j}$ is identified with a left invariant vector field on $G$. Consider the following stochastic differential equation.

$$
d g_{t}=\sum_{j=1}^{d} X_{j}\left(g_{t}\right) \circ d w_{t}^{j} .
$$

Let $g_{t}$ be the solution for which $g_{0}=e$. The process $g_{t}$ is called the horizontal diffusion in $G$. Note that the definition of $g_{t}$ depends on the choice of an orthonormal frame $u=\left(X_{1}, \ldots, X_{d}\right)$, however, its probability law is independent of $u$.

Lemma 1. Let $g_{t}$ be the horizontal diffusion in $G$ defined with respect to an orthonormal frame $u$. We have

$$
\forall g \in G, \quad F_{t}(g(u))=\left(g g_{t}\right) u .
$$

In particular, $F_{t}(u)=g_{t}(u)$. Consequently the image $x_{t}$ of $g_{t}$ under the natural projection: $G \rightarrow V$ is Brownian motion in $V$ and the canonical stochastic flow $F_{t}$ leaves the subbundle $G_{u}$ invariant. Moreover, for any $X \in \mathscr{G} \cong T_{u} G_{u}$,

$$
\left\|D F_{t}(u) X\right\|_{c}=\left\|\operatorname{Ad}\left(g_{t}^{-1}\right) X\right\|_{c} .
$$

Proof. To prove (5), it suffices to show that $u_{t}=g g_{t} u$ satisfies (1). Let $I$ be the smooth function: $G \times O(V) \rightarrow O(V)$ defined by $I(g, u)=g u$.

$$
\begin{aligned}
d\left[g g_{t} u\right]=D_{g} I\left(g g_{t}, u\right) L_{g} & \circ d g_{t}=\sum_{j} D_{g} I\left(g g_{t}, u\right) X_{j}\left(g g_{t}\right) \circ d w_{t}^{j} . \\
D_{g} I\left(g g_{t}, u\right) X_{j}\left(g g_{t}\right) & =\left.D_{g} I\left(g g_{t}, u\right) \frac{d}{d s} g g_{t} e^{s X_{j}}\right|_{s=0} \\
& =\left.\frac{d}{d s} I\left(g g_{t} e^{s X_{j}}, u\right)\right|_{s=0}=\bar{X}_{j}\left(g g_{t} u\right) .
\end{aligned}
$$

The last equality above follows from the fact that for any $X, Y \in \mathscr{G}$, the parallel displacement of $Y$ along the geodesic $t \mapsto e^{t X} O$ is given by $D \exp (t X)(Y)$, see Theorem 3.3 in [3, Chapter IV]. This shows that $u_{t}=g g_{t} u$ satisfies (1) and hence proves (5). We have

$$
D F_{t}(u)(X)=\left.\frac{d}{d s}\left[e^{s X} g_{t}(u)\right]\right|_{s=0}=\left.\frac{d}{d s}\left[g_{t} \exp \left(s \operatorname{Ad}\left(g_{t}^{-1}\right) X\right)(u)\right]\right|_{s=0} .
$$

Now (6) follows from this and the fact that the canonical metric is invariant under the action of $G$.

For our example $G=S L(n)$, we may take $\left\{X_{1}, \ldots, X_{d}\right\}$ to be the following symmetric matrices: $E_{j k} / 2 \sqrt{n}, 1 \leq j<k \leq n$, and $D_{i n} / 2 \sqrt{n}$ for $1 \leq i<n$, where $E_{j k}$ is the matrix whose $(j, k)$ and $(k, j)$ entries are one and other entries are zero, and $D_{j k}$ is the diagonal matrix whose $j$ th diagonal entry is one, $k$ th diagonal entry is negative one and other entries are zero. Let $g \in S L(n)$ and $X \in \operatorname{sl}(n)$. The tangent vector to the curve $s \mapsto g e^{s X}$ at $s=0$ is $g X$ (matrix multiplication). Therefore, when $X$ is considered as a left invariant vector field on $S L(n)$, its value at $g$ is given by $g X$. The stochastic differential 
equation (4) becomes

$$
d g_{t}=\frac{1}{2 \sqrt{n}} \sum_{j<k} g_{t} E_{j k} \circ d w_{t}^{j k}+\frac{1}{2 \sqrt{n}} \sum_{i=1}^{n-1} g_{t} D_{i n} \circ d w_{t}^{i},
$$

where $\left\{w_{t}^{j k}, w_{t}^{i} ; 1 \leq j<k \leq n, 1 \leq i<n\right\}$ is an $[(n-1)(n+2) / 2]-$ dimensional Wiener process.

By Lemma 1, to determine the exponents of $F_{t}$, it suffices to compute the limiting exponential growth rate of $\left\|\operatorname{Ad}\left(g_{t}^{-1}\right) X\right\|_{c}$. Since any two norms on a vector space are equivalent, this is the same as the limiting exponential growth rate of $\left\|\operatorname{Ad}\left(g_{t}^{-1}\right) X\right\|$. The advantage of using $\|\cdot\|$ instead of $\|\cdot\|_{c}$ is that the norm $\|\cdot\|$ on $\mathscr{G}$ is $\operatorname{Ad}(G)$-invariant for compact type $G / K$ and it is $\operatorname{Ad}(K)$-invariant for noncompact type $G / K$.

Let $X_{t}=\operatorname{Ad}\left(g_{t}^{-1}\right) X$. We have $X_{0}=X$ and $X_{t}$ satisfies the following stochastic differential equation.

$$
d X_{t}=\sum_{j=1}^{d}\left[X_{t}, X_{j}\right] \circ d w_{t}^{j}
$$

To prove (8), let $J$ be the smooth function: $G \times \mathscr{G} \rightarrow \mathscr{G}$ defined by $J(g, X)=\operatorname{Ad}\left(g^{-1}\right) X$.

$$
d X_{t}=D_{g} J\left(g_{t}, X\right) \circ d g_{t}=\sum_{j} D_{g} J\left(g_{t}, X\right) X_{j}\left(g_{t}\right) \circ d w_{t}^{j} .
$$

Since $X_{j}\left(g_{t}\right)$ is the tangent of the curve

$$
s \mapsto g_{t} \exp \left(s X_{j}\right) \quad \text { at } s=0,
$$

$D_{g} J\left(g_{t}, X\right) X_{j}\left(g_{t}\right)$ is the tangent of the curve

$$
\begin{aligned}
s \mapsto J\left(g_{t} e^{s X_{j}}, X\right) & =\operatorname{Ad}\left(e^{-s X_{j}} g_{t}^{-1}\right) X=\operatorname{Ad}\left(e^{-s X_{j}}\right) X_{t} \\
& =X_{t}+s\left[X_{t}, X_{j}\right]+O\left(s^{2}\right),
\end{aligned}
$$

so it is $\left[X_{t}, X_{j}\right]$ as desired. See (4) on p. 128 of [3] for the last equality above. Our claim is proved.

Assume that $V$ is a compact type symmetric space. Since $\langle\cdot, \cdot\rangle$ is an $\operatorname{Ad}(G)$ invariant inner product on $\mathscr{G},\langle[Z, X], Y\rangle+\langle X,[Z, Y]\rangle=0$ for $X, Y, Z \in$ $\mathscr{G}$. Hence,

$$
d\left\langle X_{t}, X_{t}\right\rangle=2 \sum_{j}\left\langle X_{t},\left[X_{t}, X_{j}\right]\right\rangle \circ d w_{t}^{j}=-2 \sum_{j}\left\langle\left[X_{t}, X_{t}\right], X_{j}\right\rangle \circ d w_{t}^{j}=0 .
$$

So $t \rightarrow\left\langle X_{t}, X_{t}\right\rangle$ is constant. It follows that $F_{t}$ restricted to $G_{u}$ has only zero exponents. In fact, this is also true for the flow $F_{t}$ on $O(V)$. To see this, note that $F_{t}(u)$ depends on the Brownian path $w$. We will write $F_{t}(u, w)$ for $F_{t}(u)$ when we wish to indicate this dependence. Any orthogonal matrix $a$ transforms $u$ into a new frame $u a$. We have $F_{t}(u a, w)=F_{t}(u, a w) a$, see [2]. Hence, by the definition of the canonical metric, if $X \in T_{u} O(V)$ and if $X$ is orthogonal to $T_{u} G_{u}$, the length of $D F_{t}(\cdot, w) X$ is no less than that of $X$. We see that if $F_{t}$ has a nonzero exponent on $O(V)$, then it has to be positive. But this is impossible since the mean exponent is zero [2]. Therefore, the flow $F_{t}$ on $O(V)$ has only zero exponent. 
Theorem 1. The canonical stochastic flow on the orthonormal frame bundle of a Riemannian symmetric space of compact type has only zero Lyapunov exponent.

\section{NONCOMPACT TYPE SYMMETRIC SPACES}

Throughout the rest of the paper, we will assume that $V=G / K$ is a $d$ dimensional Riemannian symmetric space of noncompact type with standard metric and $o$ is the coset $K$. By the general theory [3], $V$ is diffeomorphic to $R^{d}$ and has nonpositive sectional curvatures. Recall that $\mathscr{P}$ can be identified with $T_{o} V$ via the map: $\mathscr{P} \rightarrow T_{o} V$ defined by $\left.X \mapsto \frac{d}{d s} \exp (s X)\right|_{s=0}$. Since $T_{o} p V$ can be identified with $V$ via the exponential map at $o$, we see that $\mathscr{P}$ and $V$ can also be identified. We will use these identifications without further comments. Note that if $x \in V$ is identified with $X \in \mathscr{P}$, then for any $k \in K$, $k(x)$ is identified with $\operatorname{Ad}(k) X$, therefore, with identification of $V$ and $\mathscr{P}$, we should also identify the action of $K$ on $V$ and the adjoint action of $K$ on $\mathscr{P}$.

Recall that $\langle X, Y\rangle$ defined by $-B(X, \theta(Y))$ is an inner product on $\mathscr{G}$.

Let $\mathscr{A}$ be a maximal abelian subspace of $\mathscr{P}$. Here, the term "abelian" means that $[X, Y]=0$ for all $X, Y \in \mathscr{A}$. A linear functional $\alpha$ on $\mathscr{A}$ is called a root if the space

$$
\mathscr{G}_{\alpha}=\{X \in \mathscr{G} ; \forall H \in \mathscr{A}, \operatorname{ad}(H) X=\alpha(H) X\}
$$

is nonzero. $\mathscr{G}_{\alpha}$ will be called the root space of $\alpha$. It is clear that 0 is a root and its root space $\mathscr{G}_{0}=\mathscr{A} \oplus \mathscr{M}$, where $\mathscr{M}$ is the centralizer of $\mathscr{A}$ in $\mathscr{K}$, i.e., $\mathscr{M}=\{X \in \mathscr{K} ;[X, H]=0$ for $H \in \mathscr{A}\}$. We have

$$
\theta\left(\mathscr{G}_{\alpha}\right) \subset \mathscr{G}_{-\alpha} \text { and }\left[\mathscr{G}_{\alpha}, \mathscr{G}_{\beta}\right] \subset \mathscr{G}_{\alpha+\beta} .
$$

The first relation above follows from the fact that $\theta$ is a Lie algebra automorphism, $\theta(X)=X$ for $X \in \mathscr{K}$ and $\theta(X)=-X$ for $X \in \mathscr{P}$. The second relation is an easy consequence of the Jacobi identity.

For $H \in \mathscr{A}, \operatorname{ad}(H): \mathscr{G} \rightarrow \mathscr{G}$ is symmetric with respect to the inner product $\langle\cdot, \cdot\rangle$. The family of linear maps $\{\operatorname{ad}(H) ; H \in \mathscr{A}\}$ is commutative, hence, by simultaneous diagonalization, we have the following decomposition of $\mathscr{G}$ which is orthogonal with respect to $\langle\cdot, \cdot\rangle$.

$$
\mathscr{G}=\sum_{\alpha \in \Sigma} \mathscr{G}_{\alpha} \oplus \mathscr{G}_{0}
$$

where $\Sigma$ is the set of all nonzero roots. Note that our definition of roots and the above root space decomposition depend on the choice of a maximal abelian subspace $\mathscr{A}$ of $\mathscr{P}$.

Any nonzero root $\alpha$ determines a hyperplane $\alpha=0$ in $\mathscr{A}$ and $\mathscr{A}$ minus these hyperplanes consists of several open components. These components are called Weyl chambers. Now fix a Weyl chamber $\mathscr{A}^{+}$contained in $\mathscr{A}$. A root $\alpha$ is said to be positive if $\alpha>0$ on $\mathscr{A}^{+}$. Let $\Sigma^{+}$be the set of all positive roots. We have $\Sigma=\Sigma^{+} \cup\left\{-\Sigma^{+}\right\}$.

For any $\alpha \in \Sigma^{+}$, there is a unique element $H_{\alpha} \in \mathscr{A}$ such that $\forall H \in \mathscr{A}$, $\alpha(H)=B\left(H, H_{\alpha}\right)$. We will call $H_{\alpha}$ the vector representing $\alpha$. Let $m_{\alpha}=$ $\operatorname{dim}\left(\mathscr{G}_{\alpha}\right), \rho=\frac{1}{2} \sum_{\alpha \in \Sigma^{+}} m_{\alpha} \alpha$ and let $H_{\rho}$ be the vector representing $\rho$, i.e.,

$$
H_{\rho}=\frac{1}{2} \sum_{\alpha \in \Sigma^{+}} m_{\alpha} H_{\alpha} .
$$


It can be shown (see [6] or [12]) that

$$
H_{\rho} \in \mathscr{A}^{+} \text {. }
$$

Recall that for our example $G=S L(n), \mathscr{G}=s l(n), \mathscr{K}=o(n)$ and $\mathscr{P}$ is the space of symmetric matrices of trace zero. Let $\mathscr{A}$ be the space of diagonal matrices of trace zero. Then $\mathscr{A}$ is a maximal abelian subspace of $\mathscr{P}$. For a diagonal matrix $H=\operatorname{diag}\left\{\lambda_{1}, \ldots, \lambda_{d}\right\}$, let $\alpha_{j k}(H)=\lambda_{j}-\lambda_{k}$ for $j \neq k$. The set of nonzero roots is

$$
\Sigma=\left\{\alpha_{j k} ; 1 \leq j, k \leq n\right\} .
$$

Let $\mathscr{G}_{j k}$ be the root space of $\alpha_{j k}$. Then $\mathscr{G}_{j k}$ is 1-dimensional and is generated by the matrix whose $(j, k)$-entry is one and other entries are zero. Note that for our example, $\mathscr{M}=\{0\}$, so $\mathscr{G}_{0}=\mathscr{A}$. A diagonal matrix in $\mathscr{A}$ is contained in one of the hyperplanes $\alpha_{j k}=0$ if and only if at least two of its diagonal entries are the same.

Let $\mathscr{A}^{+}$be the space of diagonal matrices in $\mathscr{A}$ whose diagonal entries are in strictly descending order. Then $\mathscr{A}^{+}$is a Weyl chamber. The corresponding set of positive roots is given by

$$
\Sigma^{+}=\left\{\alpha_{j k} ; 1 \leq j<k \leq n\right\} .
$$

The vector representing $\alpha_{j k}$ is $H_{j k}=\frac{1}{2 n} D_{j k}$ (recall that $D_{j k}$ is a diagonal matrix whose $j$ th diagonal entry is one, $k$ th diagonal entry is negative one and other entries are zero) and

$$
H_{\rho}=\frac{1}{2} \sum_{j<k} H_{j k}=\frac{1}{4 n} \operatorname{diag}\{n-1, n-3, \ldots,-(n-3),-(n-1)\} .
$$

Note that (12) holds for our example.

Any element of $\mathscr{P}$ is contained in some maximal abelian subspace of $\mathscr{P}$. In fact, for any $k \in K, \operatorname{Ad}(k) \mathscr{A}$ is also a maximal abelian subspace and $\bigcup_{k \in K} \operatorname{Ad}(k) \mathscr{A}=\mathscr{P}$. For our example $G=S L(n)$, this is an easy consequence of the fact that any symmetric matrix can be diagonalized through an orthogonal transformation.

If $C$ and $C^{\prime}$ are two Weyl chambers, possibly contained in two different maximal abelian subspaces of $\mathscr{P}$, then either $C=C^{\prime}$ or $C \cap C^{\prime}$ is empty. Moreover, there exists $k \in K$ such that $\operatorname{Ad}(k) C=C^{\prime}$. An element of $\mathscr{P}$ is said to be regular if it is contained in some Weyl chamber. Let $\mathscr{P}^{\prime}$ be the set of regular elements. $\mathscr{P}^{\prime}$ is an open subset of $\mathscr{P}$ and $\mathscr{P}^{\prime}=\bigcup_{k \in K} \operatorname{Ad}(k) C$, where $C$ is a Weyl chamber. For our example $G=S L(n), \mathscr{P}^{\prime}$ consists of symmetric matrices of trace zero having distinct eigenvalues.

Let $M$ be the subgroup of $K$ whose adjoint action fixes $\mathscr{A}$, i.e.,

$$
M=\{k \in K ; \operatorname{Ad}(k) H=H, H \in \mathscr{A}\} .
$$

The Lie algebra of $M$ is $\mathscr{M}$, the centralizer in $\mathscr{K}$ of $\mathscr{A}$. The map $\Phi:(K / M) \times$ $\mathscr{A}^{+} \rightarrow \mathscr{P}^{\prime}$ defined by $\Phi(k M, H)=\operatorname{Ad}(k) H$ is a diffeomorphism (see [3, Chapter IX]). Note that for $G=S L(n)$, the identity component of $M$ is $\{e\}$.

The following lemma will be useful when studying the limiting behavior of Brownian motion in $V$. It was proved and used in [12]. 
Lemma 2. The set $\mathscr{P}-\mathscr{P}^{\prime}$ of irregular elements is contained in the union of a finitely many submanifolds of $\mathscr{P}$ whose codimensions in $\mathscr{P}$ are at least two.

Proof. For any nonzero root $\alpha$, let $\Gamma_{\alpha}$ be the hyperplane $\alpha=0$ in $\mathscr{A}$ and let $M_{\alpha}$ be the Lie subgroup of $K$ whose adjoint action fixes the elements in $\Gamma_{\alpha}$. The Lie algebra of $M_{\alpha}$ is

$$
\mathscr{M}_{\alpha}=\left\{X \in \mathscr{K} ;[X, Y]=0 \text { for } Y \in \Gamma_{\alpha}\right\} .
$$

It suffices to prove

$$
\forall \alpha \in \Sigma, \quad \operatorname{dim}\left(\operatorname{Ad}(K) \Gamma_{\alpha}\right) \leq d-2 .
$$

It is clear that $\mathscr{M} \subset \mathscr{M}_{\alpha}$. Let $X \in \mathscr{G}_{\alpha}$. There are $Y \in \mathscr{P}$ and $Z \in \mathscr{K}$ such that $X=Y+Z$. Since $\operatorname{ad}(H) X=0$ for $H \in \Gamma_{\alpha}$, we see that $\operatorname{ad}(H) Z=0$ for $H \in \Gamma_{\alpha}$. It follows that $\operatorname{dim}(\mathscr{M})<\operatorname{dim}\left(\mathscr{M}_{\alpha}\right)$, so $\operatorname{dim}\left(K / M_{\alpha}\right)<\operatorname{dim}(K / M)$. Since

$$
\operatorname{dim}(K / M)+\operatorname{dim}(\mathscr{A})=\operatorname{dim}\left(K / M \times \mathscr{A}^{+}\right)=\operatorname{dim}(\mathscr{P})=d,
$$

we see that

$$
\operatorname{dim}\left(\operatorname{Ad}(K) \Gamma_{\alpha}\right)=\operatorname{dim}\left(K / M_{\alpha} \times \Gamma_{\alpha}\right)=\operatorname{dim}\left(K / M_{\alpha}\right)+\operatorname{dim}\left(\Gamma_{\alpha}\right) \leq d-2 .
$$

Let $S$ be the unit sphere in $\mathscr{P}$ with respect to the norm $\|\cdot\|$ and let

$$
S_{\rho}=\left\{\operatorname{Ad}(k)\left(H_{\rho} /\left\|H_{\rho}\right\|\right) ; k \in K\right\} .
$$

The set $S_{\rho}$ is an $\operatorname{Ad}(K)$-invariant subset of $S$ and its definition is independent of the choice of the Weyl chamber $\mathscr{A}^{+}$. It is also clear that $X \in \mathscr{P}$ is contained in $S_{\rho}$ if and only if $X$ is $H_{\rho} /\left\|H_{\rho}\right\|$ with respect to some Weyl chamber.

For our example $S L(n), S$ is the set of symmetric matrices $X$ of trace zero such that $2 n \sum_{j, k} X_{j k}^{2}=1$, where $X_{j k}$ is the $(j, k)$-entry of the matrix $X$, and $S_{\rho}$ is the subset of $S$ consisting symmetric matrices having eigenvalues

$$
\frac{n-1}{4 n}, \frac{n-3}{4 n}, \ldots, \frac{3-n}{4 n}, \frac{3-n}{4 n} \text {. }
$$

We now define a function $\Psi$ on $S$. For $Y \in S$, choose a maximal abelian subspace $\mathscr{A}$ of $\mathscr{P}$ which contains $Y$ and define

$$
\Psi(Y)=\frac{1}{2} \sum_{\alpha \in \Sigma^{+}} m_{\alpha}|\alpha(Y)|,
$$

where the set of positive roots $\Sigma^{+}$is defined with respect to a Weyl chamber $\mathscr{A}^{+}$contained in $\mathscr{A}$. We will see later in (18) that the definition of $\Psi$ is

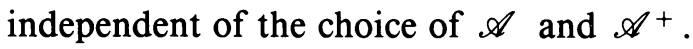

Fix $Y \in S$. Consider the symmetric bilinear form

$$
L_{Y}\left(X, X^{\prime}\right)=B\left(\operatorname{ad}(Y) X, \operatorname{ad}(Y) X^{\prime}\right)
$$

on $\mathscr{P}$. Let $\nu_{1}, \ldots, \nu_{r}$ be the distinct eigenvalues of $L_{Y}$ with the corresponding multiplicities $m_{1}^{\prime}, \ldots, m_{r}^{\prime}$. Since $\operatorname{ad}(Y) X \in \mathscr{K}$ for $X \in \mathscr{P}$ and $B$ is negative definite on $\mathscr{K}$, we see that all $\nu_{j} \leq 0$. We claim

$$
\Psi(Y)=\frac{1}{2} \sum_{i=1}^{r} m_{i}^{\prime} \sqrt{-\nu_{i}} .
$$


Choose a maximal abelian subspace $\mathscr{A}$ of $\mathscr{P}$ containing $Y$ and fix a Weyl chamber $\mathscr{A}^{+}$contained in $\mathscr{A}$. For $\alpha \in \Sigma^{+}$, let $\mathscr{P}_{\alpha}$ be the image of $\mathscr{G}_{\alpha}$ under the natural projection: $\mathscr{K} \oplus \mathscr{P} \rightarrow \mathscr{P}$. Choose an orthonormal basis $\left\{Y_{1}, \ldots, Y_{d}\right\}$ of $\mathscr{P}$ such that each $Y_{j}$ is contained either in $\mathscr{A}$ or in some $\mathscr{P}_{\alpha}$. If $Y_{j} \in \mathscr{A}, \operatorname{ad}(Y) Y_{j}=0$. If $Y_{j} \in \mathscr{P}_{\alpha}$, then there are $X_{j} \in \mathscr{G}_{\alpha}$ and $Z_{j} \in \mathscr{K}$ such that $X_{j}=Y_{j}+Z_{j}$. Since $\operatorname{ad}(Y) X_{j}=\alpha(Y) X_{j}, \operatorname{ad}(Y) Y_{j} \in \mathscr{K}$ and $\operatorname{ad}(Y) Z_{j} \in \mathscr{P}$, we see that $\operatorname{ad}(Y) Y_{j}=\alpha(Y) Z_{j}, \operatorname{ad}(Y) Z_{j}=\alpha(Y) Y_{j}$ and $\operatorname{ad}(Y)^{2} Y_{j}=\alpha(Y)^{2} Y_{j}$. It follows that

$$
L_{Y}\left(Y_{j}, Y_{k}\right)=B\left(\operatorname{ad}(Y) Y_{j}, \operatorname{ad}(Y) Y_{k}\right)=-B\left(\operatorname{ad}(Y)^{2} Y_{j}, Y_{k}\right)=-\alpha(Y)^{2} \delta_{j k} .
$$

Hence, $\left\{Y_{1}, \ldots, Y_{d}\right\}$ is a complete set of orthonormal eigenvectors of $L_{Y}$ and the eigenvalue corresponding to $Y_{j}$ is $-\alpha_{j}(Y)^{2}$, where $\alpha_{j}=0$ if $Y_{j} \in \mathscr{A}$ and $\alpha_{j}$ is the positive root $\alpha$ if $Y \in \mathscr{P}_{\alpha}$. It is now clear that the right-hand sides of (17) and (18) are the same, so (18) is proved. It follows from (18) that $\Psi$ is a continuous function on $S$.

By (17), $\Psi>0$ on $S$. For $Y \in S \cap \mathscr{A}^{+}, \Psi(Y)=B\left(Y, H_{\rho}\right)$, so $\Psi(Y) \leq$ $\left\|H_{\rho}\right\|$ and the equality holds if and only if $Y=H_{\rho} /\left\|H_{\rho}\right\|$. Since $\Psi$ is $\operatorname{Ad}(K)$ invariant by its definition, it assumes the same set of values on the closure of any Weyl chamber. This proves the following lemma.

Lemma 3. $\Psi$ defined by (18) is a positive continuous function on $S$, its maximal value is $\left\|H_{\rho}\right\|$ and $\Psi(Y)=\left\|H_{\rho}\right\|$ if and only if $Y \in S_{\rho}$.

The function $\Psi$ is naturally associated with the curvature of $V$. To see this, let $Y_{1}, \ldots, Y_{d}$ be as above. We may assume $Y_{1}=Y$. By Theorem 4.2 in [3, Chapter IV], the curvature tensor is given by $R(X, Y) Z=-[[X, Y], Z]$. Let $s_{j}$ be the sectional curvature of the section spanned by $Y$ and $Y_{j}$. Then

$$
s_{j}=\left\langle R\left(Y_{j}, Y\right) Y, Y_{j}\right\rangle=-B\left(\operatorname{ad}(Y)^{2} Y_{j}, Y_{j}\right)=\alpha_{j}(Y)^{2} .
$$

It follows that

$$
\Psi(Y)=\frac{1}{2} \sum_{j=2}^{d} \sqrt{-s_{j}} .
$$

\section{The ASYMptotic Behavior of Brownian Motion}

Let $x_{t}$ be a Brownian motion on $V$ with an arbitrary starting point and let $r_{t}=r\left(x_{t}\right)$, where $r(x)=d(o, x)$ is the Riemannian distance between $o$ and $x$. As before, we will identify $V$ with $\mathscr{P}$. Fix a maximal abelian subspace $\mathscr{A}$ of $\mathscr{P}$ and a Weyl chamber $\mathscr{A}^{+}$contained in $\mathscr{A}$. Recall that the set of regular elements, $\mathscr{P}^{\prime}$, is an open subset of $\mathscr{P}$ which is diffeomorphic to $(K / M) \times \mathscr{A}^{+}$ under the map $\Phi$. By Lemma 2 and the well-known fact that Brownian motion will never hit a set of codimension greater than or equal to two, it follows that almost surely,

$$
x_{t} \in \mathscr{P}^{\prime} \text { for } t>0 \text {. }
$$

Therefore, we always have the decomposition $x_{t}=\left(\bar{k}_{t}, A_{t}\right)$ with $\bar{k}_{t} \in K / M$ and $A_{t} \in \mathscr{A}^{+}$. Assume $m=\operatorname{dim}\left(\mathscr{A}^{+}\right)$and let $A_{1}, A_{2}, \ldots, A_{m}$ be an orthonormal basis of $\mathscr{A}$. Any $A \in \mathscr{A}^{+}$can be expressed uniquely as $A=$ $\sum_{i} a_{i} A_{i}$. Note that $\|A\|^{2}=\sum_{i} a_{i}^{2}$. We have $r_{t}=\left\|A_{t}\right\|$.

Our main objective in this section is to prove the following theorem. 
Theorem 2. Almost surely, $\left\|A_{t}\right\| \rightarrow \infty$ and $A_{t} / t \rightarrow H_{\rho}$ as $t \rightarrow \infty$, and $\lim _{t \rightarrow \infty} \bar{k}_{t}$ exists.

Recall that for $G=S L(n)$ and $K=S O(n), V=G / K$ can be identified with the space of positive definite symmetric matrices of determinant one through the map: $g \mapsto g g^{*}$ defined on $S L(n)$. Let $g_{t}$ be the horizontal diffusion in $S L(n)$ defined by (7) with $g_{0}=I$, the identity matrix, and let $x_{t}=g_{t} g_{t}^{*}$. The process $x_{t}$ in $V$ is called the Brownian motion in the space of ellipsoids by Dynkin. Let $X_{t}$ be the process in $\mathscr{P}$ such that $x_{t}=e^{X_{t}}$. Since almost surely, $X_{t} \in \mathscr{P}^{\prime}$, we see that almost surely, $x_{t}$ has distinct eigenvalues. Let $u_{t}$ be an orthonormal frame in $R^{n}$ with respect to which $x_{t}$ is diagonalized with descending diagonal entries. Such a frame is not unique because the direction of any axis of $u_{t}$ can be reversed. All such frames form an equivalence class $\bar{u}_{t}$, called a flag. It is not hard to see that the process $\bar{u}_{t}$ plays the role of $\bar{k}_{t}$ in $K / M$. Note that $x_{t}$ can be considered as an ellipsoid of unit volume and $\bar{u}_{t}$ can be considered as the axes of this ellipsoid. Now $A_{t}$ is a diagonal matrixvalued process whose diagonal entries are the eigenvalues of $X_{t}$ in descending order. The process $A_{t}$ describes the behavior of the eigenvalues of $x_{t}$. The limit $\lim _{t \rightarrow \infty} A_{t} / t=H_{\rho}$ means that for large $t$, the eigenvalues of $x_{t}$ behave like

$$
\begin{aligned}
\exp \left\{\frac{1}{4 n}(n-1) t\right\}, & \exp \left\{\frac{1}{4 n}(n-3) t\right\}, \ldots, \\
& \exp \left\{\frac{1}{4 n}(3-n) t\right\}, \exp \left\{\frac{1}{4 n}(1-n) t\right\},
\end{aligned}
$$

and the existence of the limit $\lim _{t \rightarrow \infty} \bar{k}_{t}$ means that when $t \rightarrow \infty$, the frame of the axes of the ellipsoid $x_{t}$ tends to a limit.

Lemma 4. $r_{t} \rightarrow \infty$ and $\liminf _{t \rightarrow \infty} r_{t} / t \geq c$ for some constant $c>0$.

Proof. With the polar coordinate decomposition $x_{t}=\left(r_{t}, \theta_{t}\right)$, where $\theta_{t}$ is a process in $S$, we have [9],

$$
r_{t}=r_{0}+w_{t}+\int_{0}^{t} F\left(r_{s}, \theta_{s}\right) d s,
$$

where $w_{t}$ is a 1-dimensional Wiener process and $F$ is some function on $V$. We have a useful expression for $F$ using Jacobi fields. Fix a unit vector $Y_{1}$ in $T_{0} V \cong \mathscr{P}$ and let $\gamma$ be the geodesic starting from $o$ with $\gamma^{\prime}(0)=Y_{1}$. The value of $F$ at $\gamma(r)$ is given by

$$
F=\frac{1}{2} \sum_{j=2}^{d}\left\langle\tilde{Y}_{j}^{\prime}, \tilde{Y}_{j}\right\rangle,
$$

where $\tilde{Y}_{2}, \tilde{Y}_{3}, \ldots, \tilde{Y}_{d}$ are Jacobi fields along $\gamma$ such that $\tilde{Y}_{j}(0)=0$, and $\left\{\gamma^{\prime}(r), \tilde{Y}_{2}(r), \ldots, \widetilde{Y}_{d}(r)\right\}$ form an orthonormal basis in $T_{\gamma(r)} V$.

Let $\bar{Y}_{1}, \bar{Y}_{2}, \ldots, \bar{Y}_{d}$ be the parallel displacements of $Y_{1}, Y_{2}, \ldots, Y_{d}$ along $\gamma$. By solving the Jacobi equation, we see that $\bar{Y}_{1}=\gamma^{\prime}$ and $\tilde{Y}_{j}=f_{j} \bar{Y}_{j}$, where

$$
f_{j}(t)=\frac{\sinh \left(\sqrt{-s_{j}} t\right)}{\sinh \left(\sqrt{s_{j}} r\right)}
$$


for $0 \leq t \leq r$ and $s_{j}$ is the sectional curvature of the section spanned by $Y_{1}$ and $Y_{j}$. By (22), for fixed $\theta=Y_{1} \in S$,

$$
F(r, \theta)=\frac{1}{2} \sum_{j=2}^{d} \sqrt{-s_{j}} \operatorname{coth}\left(\sqrt{-s_{j}} r\right) .
$$

It follows that $F(r, \theta) \rightarrow \sum_{i} \sqrt{-s_{i}}=\Psi(\theta)$ as $t \rightarrow \infty$ uniformly for $\theta$. Recall that $\Psi$ can be defined by (19) and $\Psi$ is a positive continuous function on $S$, so there is a constant $c>0$ such that $\Psi \geq c$. As $t \rightarrow \infty, w_{t}=O(\sqrt{t \log \log t})$, it follows from (21) that $\liminf r_{t} / t \geq c$.

Recall that $\mathscr{P}^{\prime}$ is identified with an open subset of $V$ and is diffeomorphic to $(K / M) \times \mathscr{A}^{+}$. Let $\Delta$ be the Laplace-Beltrami operator on $\mathscr{P}^{\prime}$. By Theorem 5.24 in [4, Chapter II], if $f$ is a smooth function on $\mathscr{A}^{+}$and if it is considered as a function on $\mathscr{P}^{\prime}=(K / M) \times \mathscr{A}^{+}$, then $\Delta f=\Delta_{\mathscr{A}^{+}} f$, with $\Delta_{\mathscr{A}^{+}}$the differential operator on $\mathscr{A}^{+}$defined by

$$
\Delta_{\mathscr{A}^{+}}=\Delta_{0}+\sum_{\alpha \in \Sigma^{+}} m_{\alpha} \operatorname{coth}(\alpha) H_{\alpha},
$$

where $\Delta_{0}$ is the Euclidean Laplacian on $\mathscr{A}^{+}$and the root vectors $H_{\alpha}$ are considered as vector fields on $\mathscr{A}^{+}$.

From this we can conclude that $A_{t}$ is a diffusion process on $\mathscr{A}^{+}$with generator $\Delta_{\mathscr{A}+} / 2$. Hence,

$$
A_{t}=A_{0}+W_{t}+\int_{0}^{t} F\left(A_{s}\right) d s,
$$

where $W_{t}$ is an $m$-dimensional Wiener process and the vector field $F(A)$ on $\mathscr{A}^{+}$is given by

$$
F(A)=\frac{1}{2} \sum_{\alpha \in \Sigma^{+}} m_{\alpha} \operatorname{coth}(\alpha(A)) H_{\alpha} .
$$

The limiting property of $A_{t}$ is stated in the next lemma which is due to Orihara [7]. We present a different proof here.

Lemma 5. $A_{t}$ is a diffusion process on $\mathscr{A}^{+}$with generator $\Delta_{\mathscr{A}^{+}}$defined by (23) and almost surely, $\left\|A_{t}\right\| \rightarrow \infty$ and $A_{t} / t \rightarrow H_{\rho}$ as $t \rightarrow \infty$.

Proof. We have seen that $\left\|A_{t}\right\|=r_{t} \rightarrow \infty$ as $t \rightarrow \infty$. Since $x_{t} \in \mathscr{P}^{\prime}$ for $t>0$, $A_{t}$ never hits the boundary of $\mathscr{A}^{+}$. It remains to prove that $A_{t} / t \rightarrow H_{\rho}$ as $t \rightarrow \infty$.

If we can show that $\alpha\left(A_{t}\right) \rightarrow \infty$ as $t \rightarrow \infty$ for any $\alpha \in \Sigma^{+}$, then by (25),

$$
\lim _{t \rightarrow \infty} F\left(A_{t}\right)=\frac{1}{2} \sum_{\alpha \in \Sigma^{+}} m_{\alpha} H_{\alpha}=H_{\rho} .
$$

By (24) and the fact that $W_{t}=O(\sqrt{t \log \log t})$ as $t \rightarrow \infty$,

$$
\frac{A_{t}}{t}=\frac{A_{0}}{t}+\frac{W_{t}}{t}+\frac{1}{t} \int_{0}^{t} F\left(A_{s}\right) d s \rightarrow H_{\rho} .
$$

Therefore, it suffices to show that $\alpha\left(A_{t}\right) \rightarrow \infty$ as $t \rightarrow \infty$ for any $\alpha \in \Sigma^{+}$.

For any $A \in \mathscr{A}^{+}$with $r=\|A\|$, let $\phi(A)$ be the angle between $A$ and $H_{\rho}$, considered as vectors at the origin. Recall that $H_{\rho} \in \mathscr{A}^{+}$. We can introduce the 
polar coordinates $\left(r, \phi, \psi_{1}, \ldots, \psi_{m-2}\right)$ on $\mathscr{A}^{+}$such that $r=\|A\|, \phi=\phi(A)$ and $\left(\phi, \psi_{1}, \ldots, \psi_{m-2}\right)$ are orthogonal coordinates on the unit sphere $S$. For any $\alpha \in \Sigma^{+}$, let $\phi_{\alpha}(A)$ be the angle between $A$ and the hyperplane $\alpha=0$. It is clear that for sufficiently small $\varepsilon>0$, if $\phi(A) \leq \varepsilon$ when $\|A\|$ is sufficiently large, then $\alpha(A) \rightarrow \infty$ for any $\alpha \in \Sigma^{+}$. Therefore, in order to prove Lemma 5, it suffices to show that $\forall \varepsilon>0, \phi\left(A_{t}\right) \leq 2 \varepsilon$ for sufficiently large $t$.

$\phi$ can be considered as a function on $\mathscr{A}^{+}$, and $H_{\rho}, H_{\alpha}$ and $F(A)$ as vector fields. A direct computation using polar coordinates shows that $H_{\alpha} \phi=$ $O(1 / r)$ as $r \rightarrow \infty$. Since $H_{\rho}$ is at the north pole, one can check that $H_{\rho} \phi=$ $-\left\|H_{\rho}\right\| \sin \phi / r$. On the hyperplane $\alpha=0$, the vector $H_{\alpha}$ is in the inward normal direction with respect to $\mathscr{A}^{+}$, therefore, $H_{\alpha} \phi \leq 0$ if $\phi_{\alpha}(A)$ is sufficiently small. By (25),

$$
F \phi=H_{\rho} \phi+\frac{1}{2} \sum_{\alpha \in \Sigma^{+}} m_{\alpha}[\operatorname{coth}(\alpha(A))-1] H_{\alpha} \phi .
$$

Note that $\operatorname{coth}(\alpha(A)) \approx 1$ when $\alpha(A)$ is large, which is the case if $\|A\|$ is large and $\phi_{\alpha}(A)$ is not too small. On the other hand, if $\phi_{\alpha}(A)$ is small, $H_{\alpha} \phi \leq 0$. Therefore, by the above expression for $F \phi$, we can show that $\forall \varepsilon>0, \exists C, c>$ 0 such that

$$
F \phi \leq-\frac{c}{r} \text { if } A \in \mathscr{A}^{+} \text {with } \phi(A)>\varepsilon \text { and }\|A\| \geq C .
$$

We would like to apply Ito's formula to $\phi$. However, $\phi$ is not smooth at $\phi=0$. Let $f(x)$ be a positive smooth function on $[0, \infty)$ such that $f(x)=x$ for $x>\varepsilon, f(x) \leq \varepsilon$ for $x \leq \varepsilon$ and $f^{\prime}(x)=0$ for $x \leq \varepsilon / 2$. Set $\psi=f(\phi)$. Then $\psi$ is a smooth function on $\mathscr{A}^{+}$and $\psi=\phi$ on $[\phi>\varepsilon]$. Moreover, $\psi>\varepsilon$ if and only if $\phi>\varepsilon$. It suffices to show $\psi\left(A_{t}\right) \leq 2 \varepsilon$ for sufficiently large $t$.

By Ito's formula,

$$
\psi\left(A_{t}\right)=\psi\left(A_{0}\right)+M_{t}+\frac{1}{2} V_{t}+\int_{0}^{t}(F \psi)\left(A_{s}\right) d s,
$$

where $M_{t}=\int_{0}^{t} \nabla \psi\left(A_{s}\right) d W_{s}, V_{t}=\int_{0}^{t} \Delta \psi\left(A_{s}\right) d s$ and the gradient $\nabla$ and the Laplacian $\Delta$ are computed with respect to the Euclidean metric.

$M_{t}$ is a martingale whose bracket $\langle M\rangle_{t}=\int_{0}^{t}\left|\nabla \psi\left(A_{s}\right)\right|^{2} d s$. Since $\nabla \phi=$ $O(1 / r), \nabla \psi=f^{\prime}(\phi) \nabla \phi$ and $r_{t}=O(t)$ as $t \rightarrow \infty$, we see that

$$
\left|\nabla \psi\left(A_{s}\right)\right|^{2}=O\left(r_{s}^{-2}\right)=O\left(s^{-2}\right) .
$$

This implies that $\langle M\rangle_{\infty}<\infty$ and hence, almost surely, $M_{t}$ has a finite limit as $t \rightarrow \infty$. Recall $m=\operatorname{dim}\left(\mathscr{A}^{+}\right)$. The case when $m=1$ is trivial. We will assume $m \geq 2$. One can show that $\Delta \phi=0$ if $m=2$ and $|\Delta \phi| \leq c / r^{m-1} \sin \phi$ for some constant $c>0$ if $m>2$. Since

$$
\Delta \psi=f^{\prime \prime}(\phi)|\nabla \phi|^{2}+f^{\prime}(\phi) \Delta \phi,
$$

we see that $\Delta \psi=O\left(r^{-2}\right)$ if $m>2$. It follows that $\int_{0}^{\infty}\left|\Delta \psi\left(A_{s}\right)\right| d s$ is finite and $V_{t}$ has a finite limit as $t \rightarrow \infty$.

We claim that $\forall T>0, \exists t \geq T$ such that $\psi\left(A_{t}\right) \leq \varepsilon$. Otherwise, for some $T>0$, we would have $\psi\left(A_{t}\right)>\varepsilon$ for $t \geq T$. Because (26) holds when $\phi$ is replaced by $\psi, \int_{0}^{t}(F \psi)\left(A_{s}\right) d s$ would tend to $-\infty$ as $t \rightarrow \infty$, and by (27), 
$\psi\left(A_{t}\right) \rightarrow-\infty$. This is impossible, so our claim is true. Since both $M_{t}$ and $V_{t}$ have finite limits as $t \rightarrow \infty$, and $F \psi(A) \leq 0$ when $\psi(A)>\varepsilon$ and $\|A\|$ is large, it follows from (26) and (27) that if $\psi\left(A_{t}\right) \leq \varepsilon$ for some large $t$, then $\psi\left(A_{t}\right) \leq 2 \varepsilon$ as $t \rightarrow \infty$. This concludes our proof of Lemma 5 .

Recall $\Sigma^{+}$is the set of positive roots. For $\alpha \in \Sigma^{+}$, let $\mathscr{P}_{\alpha}$ and $\mathscr{K}_{\alpha}$ be, respectively, the images of $\mathscr{G}_{\alpha}$ under the natural projections of $\mathscr{P} \oplus \mathscr{K}$ onto $\mathscr{P}$ and $\mathscr{K}$. We have

$$
\mathscr{P}=\mathscr{A} \bigoplus \sum_{\alpha \in \Sigma^{+}} \mathscr{P}_{\alpha} \quad \text { and } \quad \mathscr{K}=\mathscr{M} \bigoplus \sum_{\alpha \in \Sigma^{+}} \mathscr{K}_{\alpha}
$$

Let $Z_{1}, Z_{2}, \ldots, Z_{l}$ be a basis of $\sum_{\alpha \in \Sigma^{+}} \mathscr{K}_{\alpha}$, which is orthonormal with respect to $\langle\cdot, \cdot\rangle$, such that each $Z_{i}$ is contained in some $\mathscr{K}_{\alpha}$. Recall that $A_{1}, \ldots, A_{m}$ is an orthonormal basis of $\mathscr{A}$. For $(a, b)=\left(a_{1}, \ldots, a_{m}, b_{1}, \ldots, b_{l}\right) \in R^{m+l}$, define

$$
\Phi^{\prime}(a, b)=\operatorname{Ad}\left(\exp \left(\sum_{i=1}^{l} b_{i} Z_{i}\right)\right)\left(\sum_{j=1}^{m} a_{j} A_{j}\right) .
$$

For $\delta>0$, let

$$
U_{\delta}^{\prime}=\left\{Y \in \mathscr{P} ; Y=\Phi^{\prime}(a, b), \quad \sum_{i} a_{i} A_{i} \in \mathscr{A}^{+} \text {and }|b|<\delta\right\} .
$$

When $\delta$ is sufficiently small, $U_{\delta}^{\prime}$ is an open subset of $\mathscr{P}$ and $\Phi^{\prime}$ is a diffeomorphism from an open subset of $R^{m+l}$ onto $U_{\delta}^{\prime}$. Therefore, $(a, b)$ can be used as local coordinates on $U_{\delta}^{\prime}$. Note that if we allow $\mathscr{A}^{+}$to vary over all Weyl chambers, then $\left\{U_{\delta}^{\prime}\right\}$ will form an open cover of $\mathscr{P}^{\prime}$.

We will express the Riemannian metric in the coordinates $(a, b)$. Let $Z_{l+1}$, $\ldots, Z_{h}$ be a basis of $\mathscr{M}$. For a small perturbation $\Delta b$ of $b$, we have

$$
\begin{aligned}
& \exp \left(\sum_{j=1}^{l}\left(b_{j}+\Delta b_{j}\right) Z_{j}\right) \\
& \quad=\exp \left(\sum_{j=1}^{l} b_{j} Z_{j}\right) \exp \left(\sum_{i=1}^{h} \sum_{j=1}^{l} a_{i j}(b) \Delta b_{j} Z_{i}+O\left(|\Delta b|^{2}\right)\right),
\end{aligned}
$$

where $a_{i j}(b)$ is a smooth function of $b$ and $a_{i j}(0)=\delta_{i j}$.

Lemma 6. For $X \in \mathscr{A}^{+}$and $Y \in \mathscr{P}_{\alpha}$ for some $\alpha \in \Sigma^{+}$, we have $\exp (X+t Y)=$ $\exp (X) \exp \left(t Z+O\left(t^{2}\right)\right)$, where

$$
Z=\alpha(X)^{-1} \sinh (\alpha(X)) Y-\alpha(X)^{-2}[\cosh (\alpha(X))-1] \operatorname{ad}(X) Y .
$$

Proof. By Theorem 1.7 in [3, Chapter II], $\forall X, Y \in \mathscr{G}, \exp (X+t Y)=$ $\exp (X) \exp \left(t Z+O\left(t^{2}\right)\right)$, where

$$
Z=\sum_{p=0}^{\infty} \frac{(-1)^{p}}{(p+1) !} \operatorname{ad}(X)^{p} Y
$$

Now (31) follows from (32) and the identity $\operatorname{ad}(X)^{2} Y=\alpha(X)^{2} Y$. 
For each $Z_{i} \in \mathscr{K}_{\alpha}$, we can choose $Y_{i} \in \mathscr{P}_{\alpha}$ such that $Y_{i}+Z_{i} \in \mathscr{G}_{\alpha}$. Thus, $Y_{1}, \ldots, Y_{l}$ form an orthonormal basis of $\sum_{\alpha \in \Sigma^{+}} \mathscr{P}_{\alpha}$. We will let $\alpha_{i}$ be the positive root $\alpha$ if $i \leq l$ and $Z_{i} \in \mathscr{K}_{\alpha}$, and let $\alpha_{i}=0$ if $i=l+1, \ldots, h$. We have

$$
\forall H \in \mathscr{A}, \quad \operatorname{ad}(H) Z_{i}=\alpha_{i}(H) Y_{i} \quad \text { and } \quad \operatorname{ad}(H) Y_{i}=\alpha_{i}(H) Z_{i} .
$$

Lemma 7. In the coordinates $(a, b)$, the Riemannian metric is given by

$$
\begin{aligned}
& \left\langle\frac{\partial}{\partial a_{p}}, \frac{\partial}{\partial a_{q}}\right\rangle=\delta_{p q}, \quad\left\langle\frac{\partial}{\partial a_{p}}, \frac{\partial}{\partial b_{q}}\right\rangle=0, \\
& \left\langle\frac{\partial}{\partial b_{p}}, \frac{\partial}{\partial b_{q}}\right\rangle=\sum_{i=1}^{l} a_{i p}(b) a_{i q}(b) \sinh ^{2}\left(\alpha_{i}(A)\right),
\end{aligned}
$$

where $A=\sum_{j=1}^{m} a_{j} A_{j}$.

Proof. The first equality follows from the fact that the Riemannian metric is flat on $\mathscr{A}^{+}$and $\operatorname{Ad}(k)$ is an isometry for $k \in K$. By (33),

$$
\left.\frac{d}{d t} \operatorname{Ad}\left(\exp \left(t Z_{i}\right)\right) A\right|_{t=0}=\left.\frac{d}{d t} e^{t \operatorname{ad}\left(Z_{i}\right)} A\right|_{t=0}=\operatorname{ad}\left(Z_{i}\right) A=-\alpha_{i}(A) Y_{i} .
$$

Note that $Y_{i}$ as a vector at $o$ is now considered as a vector at $A$, the tangent vector of the curve $t \mapsto A+t Y_{i}$ at $t=0$. Let $\bar{Y}_{i}$ be the parallel displacement of $Y_{i}$ at $o$ along the geodesic $\gamma: t \mapsto t A$. Since $e^{A}: T_{0} V \rightarrow T_{e^{A}(o)} V$ gives the parallel displacement along $\gamma$ and by Lemma 6 ,

$$
\exp \left(A+t Y_{i}\right)=e^{A} \exp \left(t F+O\left(t^{2}\right)\right),
$$

where $F=\alpha_{i}(A)^{-1} \sinh \left(\alpha_{i}(A)\right) Y_{i}+Y^{\prime}$ for some $Y^{\prime} \in \mathscr{K}$, we see that

$$
Y_{i}=\alpha_{i}(A)^{-1} \sinh \left(\alpha_{i}(A)\right) \bar{Y}_{i} \text { at } A \text {. }
$$

Let $Z=\sum_{i} b_{i} Z_{i}$. We have, by (30),

$$
\begin{aligned}
\operatorname{Ad}\left(e^{Z+t Z_{p}}\right) A & =\operatorname{Ad}\left(e^{Z}\right) \operatorname{Ad}\left(\exp \left(t \sum_{i} a_{i p}(b) Z_{i}+O\left(t^{2}\right)\right)\right) A \\
& =\operatorname{Ad}\left(e^{Z}\right) e^{t \sum_{i} a_{i p}(b) \operatorname{ad}\left(Z_{i}\right)+O\left(t^{2}\right)} A
\end{aligned}
$$

and by (33),

$$
\begin{aligned}
\operatorname{Ad}\left(e^{Z+t Z_{p}}\right) A & =\operatorname{Ad}\left(e^{Z}\right)\left[A-t \sum_{i} a_{i p}(b) \alpha_{i}(A) Y_{i}+O\left(t^{2}\right)\right] \\
\frac{\partial}{\partial b_{p}} & =\operatorname{Ad}\left(e^{Z}\right)\left[-\sum_{i} a_{i p}(b) \sinh \left(\alpha_{i}(A)\right) \bar{Y}_{i}\right]
\end{aligned}
$$

Since $\operatorname{Ad}\left(e^{Z}\right)$ is an isometry, we obtain the desired formulae in Lemma 7.

Let $\gamma$ be a curve contained in $U_{\delta}^{\prime}$ and let $d s$ be the differential of arclength on $\gamma$. By Lemma 7 ,

$$
d s^{2}=\sum_{i} d a_{i}^{2}+\sum_{i, p, q} a_{i p}(b) a_{i q}(b) \sinh ^{2}\left(\alpha_{i}(A)\right) d b_{p} d b_{q} .
$$


Let

$$
U_{\delta}=\left\{Y \in \mathscr{P} ; \quad Y=\Phi^{\prime}(a, b),|b|<\delta,\|A\| \geq 1 \text { and } \phi(A)<\delta\right\} .
$$

Recall that $A=\sum_{i} a_{i} A_{i}$ and $\phi(A)$ is the angle between $A$ and $H_{\rho}$. It is clear that $U_{\delta}$ is an open subset of $U_{\delta}^{\prime}$. Note that if $Y=\Phi^{\prime}(a, b)$, then $\|A\|=d(o, Y)$.

Lemma 8. $\forall \varepsilon>0, \exists \delta>0$ and $C>0$ such that along any curve $\gamma$ contained in $U_{\delta},|d b| \leq C \exp \left(-(1-\varepsilon) \lambda r /\left\|H_{\rho}\right\|\right) d s$, where $r=d(o, \gamma)$, the Riemannian distance between $o$ and $\gamma$, and $\lambda=\min _{\alpha \in \Sigma^{+}} \alpha\left(H_{\rho}\right)$.

Proof. For any $\varepsilon>0$, when $\|A\| \geq 1$ and when $\phi(A)$ is sufficiently small, $A \approx H_{\rho}\|A\| /\left\|H_{\rho}\right\|$, so

$$
\sinh (\alpha(A)) \geq c \exp \left((1-\varepsilon) \alpha\left(H_{\rho}\right)\|A\| /\left\|H_{\rho}\right\|\right),
$$

for some constant $c>0$. Since $a_{i p}(b)$ is continuous in $b$ and $a_{i p}(0)=\delta_{i p}$, the smallest eigenvalue of the symmetric matrix $\sum_{i} a_{i p}(b) a_{i q}(b)$ is bounded from below by a positive constant. Lemma 8 follows from (34).

Lemma 9. Almost surely, $\exists$ an integer $N>0$ and $0<\eta<1$ such that $\forall$ integer $n \geq N$,

$$
\sup \left\{d\left(x_{t}, x_{n}\right) ; n \leq t \leq n+1\right\} \leq n^{\eta} .
$$

The above formula is given in [10] (see line 8 on p. 1541, where $p^{\gamma}$ should read $\left.p^{j}\right)$. In that paper, it was assumed that the sectional curvatures were bounded from above and below by two negative numbers. However, a careful examination of the proof of this formula reveals that the boundedness of sectional curvatures was only used to control the function $F$ in (21), where the boundedness of Ricci curvature suffices. Therefore, this formula holds also for a symmetric space of noncompact type.

Now, we are ready to prove Theorem 2. By Lemma 5, it suffices to show that almost surely, $\exists$ an integer $N$ such that if $x_{N} \in U_{\delta / 2}$ for some Weyl chamber $\mathscr{A}^{+}$and $\delta>0$, then $\left|b\left(x_{t}\right)\right|<\delta$ for $t \geq N$ and $\lim _{t \rightarrow \infty} b\left(x_{t}\right)$ exists.

Choose a typical path $w$. Fix $\varepsilon>0$ and let $N$ be the integer in Lemma 9. By Lemma $5, \lim _{t \rightarrow \infty} r_{t} / t=\left\|H_{\rho}\right\|$, and so we may assume $(1-\varepsilon)\left\|H_{\rho}\right\| \leq r_{t} / t$ for $t \geq N$. For $n \geq N$, let $\gamma_{n}$ be the minimal geodesic joining $x_{n}$ and $x_{n+1}$. Since the length of $\gamma_{n}$ is at most $n^{\eta}$,

$$
d\left(o, \gamma_{n}\right) \geq d\left(o, x_{n}\right)-n^{\eta}=r_{n}-n^{\eta} \geq(1-\varepsilon)\left\|H_{\rho}\right\| n-n^{\eta} .
$$

$N$ can be chosen to be large enough so that

$$
\sum_{n \geq N} C \exp \left[-(1-\varepsilon) \lambda\left((1-\varepsilon)\left\|H_{\rho}\right\| n-n^{\eta}\right) /\left\|H_{\rho}\right\|\right] n^{\eta}<\frac{\delta}{2},
$$

where $\delta$ is the constant in Lemma 8. Assume $x_{N} \in U_{\delta / 2}$. We have $\left|b\left(x_{N}\right)\right|<$ $\delta / 2$ and by Lemma 8

$$
\sum_{n \geq N}\left|b\left(x_{n+1}\right)-b\left(x_{n}\right)\right| \leq \sum_{n \geq N} \int_{\gamma_{n}}|d b|<\frac{\delta}{2} .
$$

So $\left\{b\left(x_{n}\right)\right\}$ is a Cauchy sequence and $\left|b\left(x_{n}\right)\right|<\delta$. For $n \leq t \leq n+1$, let $\gamma^{\prime}$ be the minimal geodesic joining $x_{n}$ and $x_{t}$. Then

$$
\left|b\left(x_{t}\right)-b\left(x_{n}\right)\right| \leq \int_{\gamma^{\prime}}|d b| \leq C \exp \left[-(1-\varepsilon) \lambda\left((1-\varepsilon)\left\|H_{\rho}\right\| n-n^{\eta}\right) /\left\|H_{\rho}\right\|\right] n^{\eta} .
$$


Hence, $\left|b\left(x_{t}\right)\right|<\delta$ for $t \geq N$ and $\lim _{t \rightarrow \infty} b\left(x_{t}\right)$ exists. This concludes the proof of Theorem 2 .

As a direct consequence of Theorem 2, we obtain

Corollary 1. Let $x_{t}=\left(r_{t}, \theta_{t}\right)$ be the polar coordinate decomposition of the Brownian motion $x_{t}$ about $o$ with $\theta_{t} \in S$. Then, almost surely, $\lim _{t \rightarrow \infty} r_{t} / t=\left\|H_{\rho}\right\|$, and $\lim _{t \rightarrow \infty} \theta_{t}$ exists and is contained in $S_{\rho}$.

Note that if $x_{0}=o$, then $\theta_{\infty}=\lim _{t \rightarrow \infty} \theta_{t}$ is uniformly distributed on $S_{\rho}$ in the sense that its distribution is $\operatorname{Ad}(K)$-invariant.

\section{THE ASYMPTOTIC STABILITY OF THE CANONICAL STOCHASTIC FLOW}

We will continue to use the notations introduced earlier. Fix an orthonormal frame $u=\left(X_{1}, \ldots, X_{d}\right)$ at $o$. In $\S 2$, we have seen that to determine the Lyapunov exponents of the canonical stochastic flow $F_{t}$ on $G_{u}$, it suffices to determine the exponential growth rate of $\left\|\operatorname{Ad}\left(g_{t}^{-1}\right) X\right\|$ for any $X \in \mathscr{G}$, where $g_{t}$ is the horizontal diffusion in $G$ defined with respect to the frame $u$. Recall that the norm $\|\cdot\|$ is $\operatorname{Ad}(K)$-invariant on $\mathscr{G}$.

By Lemma 1, the image $x_{t}$ of $g_{t}$ under the natural projection: $G \rightarrow V$ is Brownian motion in $V$. By Theorem 1.1 in [3, Chapter VI], the map: $\mathscr{P} \times K \rightarrow G$ defined by $(X, k) \mapsto e^{X} k$ is a diffeomorphism, so with the identification of $V$ with $\mathscr{P}, g_{t}=\exp \left(x_{t}\right) k_{t}$ for some process $k_{t}$ on $K$. With polar decomposition, $x_{t}=r_{t} \theta_{t}$, we may write $g_{t}=\exp \left(r_{t} \theta_{t}\right) k_{t}$ and

$$
\left\|\operatorname{Ad}\left(g_{t}^{-1}\right) W\right\|=\left\|\operatorname{Ad}\left(k_{t}^{-1}\right) \operatorname{Ad}\left(e^{-r_{t} \theta_{t}}\right) W\right\|=\left\|e^{-r_{t} \operatorname{ad}\left(\theta_{t}\right)} W\right\| .
$$

Note that almost surely, $\theta_{\infty}=\lim _{t \rightarrow \infty} \theta_{t}$ exists and is contained in $S_{\rho}$. Therefore, there is a Weyl chamber $\mathscr{A}^{+}$such that $\theta_{\infty} \in \mathscr{A}^{+}$and $\theta_{\infty}=H_{\rho} /\left\|H_{\rho}\right\|$.

Theorem 3. Assume that $\theta_{\infty}$ is contained in $\mathscr{A}^{+}$. If $W \in \mathscr{G}_{\beta}$ with $W \neq 0$, where $\beta$ is a root, then

$$
\lim _{t \rightarrow \infty}\left\|\operatorname{Ad}\left(g_{t}^{-1}\right) W\right\|^{1 / t}=e^{-\beta\left(H_{\rho}\right)}
$$

Proof. Since $\theta_{t} \approx \theta_{\infty}$ for large $t$ and $\operatorname{ad}\left(\theta_{\infty}\right) W=\beta\left(\theta_{\infty}\right) W$, it may seem that (36) can be derived as follows.

$$
\left\|e^{-r_{t} \operatorname{ad}\left(\theta_{t}\right)} W\right\|^{1 / t} \approx\left\|e^{-r_{t} \operatorname{ad}\left(\theta_{\infty}\right)} W\right\|^{1 / t}=e^{-\beta\left(\theta_{\infty}\right) r_{t} / t}\|W\|^{1 / t}
$$

which tends to $\exp \left(-\beta\left(\theta_{\infty}\right)\left\|H_{\rho}\right\|\right)$ as $t \rightarrow \infty$. However, the above $\approx$ cannot be easily justified. This forces us to use a more careful argument.

We may assume that $x_{t} \in U_{\delta}$ for sufficiently large $t, x_{t}=\operatorname{Ad}\left(h_{t}\right) A_{t}$, where $h_{t}=\exp \left(\sum_{p=1}^{l} b_{p}\left(x_{t}\right) Z_{p}\right)$ and $A_{t} \in \mathscr{A}^{+}$, and $A_{t} / t \rightarrow H_{\rho}$ and $b_{p}\left(x_{t}\right) \rightarrow 0$ as $t \rightarrow \infty$. Recall that $Z_{1}, \ldots, Z_{l}$ is a basis of $\sum_{\alpha \in \Sigma^{+}} \mathscr{K}_{\alpha}$ such that each $Z_{i}$ is contained in some $\mathscr{K}_{\alpha}$. We have

$$
\begin{aligned}
\left\|\operatorname{Ad}\left(g_{t}^{-1}\right) W\right\| & =\left\|\operatorname{Ad}\left(e^{-x_{t}}\right) W\right\|=\left\|e^{-\operatorname{ad}\left[\operatorname{Ad}\left(h_{t}\right) A_{t}\right]} W\right\| \\
& =\left\|\operatorname{Ad}\left(h_{t}\right) e^{-\operatorname{ad}\left(A_{l}\right)} \operatorname{Ad}\left(h_{t}^{-1}\right) W\right\|=\left\|e^{-\operatorname{ad}\left(A_{t}\right)} \operatorname{Ad}\left(h_{t}^{-1}\right) W\right\| .
\end{aligned}
$$

Let $W_{1}, \ldots, W_{n}$ be a basis of $\mathscr{G}$ such that each $W_{i}$ is contained in $\mathscr{G}_{\beta}$ for some root $\beta$. We will use $\beta_{i}$ to denote the root $\beta$ if $W_{i} \in \mathscr{G}_{\beta}$. We may assume $W=W_{j}$ for some $j$. We have

$$
\operatorname{Ad}\left(e^{-\sum_{p=1}^{l} \sum b_{p} Z_{p}}\right) W_{j}=\sum_{k=1}^{n} f_{j k}(b) W_{k} .
$$


It is clear that the $f_{j k}(b)$ are bounded smooth functions and $f_{j k}(0)=\delta_{j k}$. We have

$$
\begin{aligned}
e^{-a d\left(A_{t}\right)} \operatorname{Ad}\left(h_{t}^{-1}\right) W_{j} & =e^{-\operatorname{ad}\left(A_{t}\right)} \operatorname{Ad}\left(e^{-\sum_{p} b_{p}\left(x_{t}\right) Z_{p}}\right) W_{j} \\
& =\sum_{k=1}^{n} f_{j k}\left(b\left(x_{t}\right)\right) e^{-\beta_{k}\left(A_{t}\right)} W_{k} .
\end{aligned}
$$

Note that $f_{j k}\left(b\left(x_{t}\right)\right) \rightarrow \delta_{j k}$ and $\beta_{k}\left(A_{t}\right)=t \beta_{k}\left(A_{t} / t\right) \approx t \beta_{k}\left(H_{\rho}\right)$ as $t \rightarrow \infty$, so the $j$ th term of the right-hand side of (38) has the correct exponential growth rate for (36) with $W=W_{j}$. To prove (36), we need to show that the exponential growth rates of the other terms are not greater.

The following lemma will be proved in the next section.

Lemma 10. If $\beta_{j} \neq \beta_{k}$, then $\forall \varepsilon>0$,

$$
\left|f_{j k}\left(b\left(x_{t}\right)\right)\right| \leq e^{-(1-\varepsilon)\left|\beta_{j}\left(H_{\rho}\right)-\beta_{k}\left(H_{\rho}\right)\right| t},
$$

for sufficiently large $t>0$.

We now show that Theorem 3 follows from the above lemma. Note that the sum on the right-hand side of (38) is orthogonal, we have

$$
\begin{gathered}
\left\|\operatorname{Ad}\left(g_{t}^{-1}\right) W_{j}\right\|^{2}=\left\|e^{-\operatorname{ad}\left(A_{t}\right)} \operatorname{Ad}\left(h_{t}^{-1}\right) W_{j}\right\|^{2}=\sum_{k}\left|f_{j k}\left(b\left(x_{t}\right)\right)\right|^{2} e^{-2 \beta_{k}\left(A_{t}\right)} . \\
\liminf _{t \rightarrow \infty}\left\|\operatorname{Ad}\left(g_{t}^{-1}\right) W_{j}\right\|^{1 / t} \geq \liminf _{t \rightarrow \infty}\left|f_{j j}\left(b\left(x_{t}\right)\right)\right|^{1 / t} e^{-\beta_{j}\left(A_{t}\right) / t}=e^{-\beta_{j}\left(H_{\rho}\right)} .
\end{gathered}
$$

Since $A_{t}=H_{\rho} t+o(t)$, by Lemma 10,

$$
\left|f_{j k}\left(b\left(x_{t}\right)\right)\right| e^{-\beta_{k}\left(A_{t}\right)} \leq e^{-\beta_{j}\left(H_{\rho}\right) t+\varepsilon\left|\beta_{j}-\beta_{k}\right|\left(H_{\rho}\right) t+o(t)} .
$$

It follows that

$$
\limsup _{t \rightarrow \infty}\left\|\operatorname{Ad}\left(g_{t}^{-1}\right) W_{j}\right\|^{1 / t} \leq e^{-\beta_{j}\left(H_{\rho}\right)} \max _{k} e^{\varepsilon\left|\beta_{j}-\beta_{k}\right|\left(H_{\rho}\right)} .
$$

Since $\varepsilon>0$ can be made arbitrarily small, Theorem 3 is proved.

Let $\mu_{1}>\mu_{2}>\cdots>\mu_{s}$ be the distinct values contained in the set $\left\{\alpha\left(H_{\rho}\right) ; \alpha\right.$ $\left.\in \Sigma^{+}\right\}$. Since any nonzero root is either $\alpha$ or $-\alpha$ for some $\alpha \in \Sigma^{+}$, the eigenvalues of the linear map $\operatorname{ad}\left(H_{\rho}\right): \mathscr{G} \rightarrow \mathscr{G}$ are given by $\left\{\mu_{i} ; i=0, \pm 1, \pm 2\right.$, $\ldots, \pm s\}$, where $\mu_{0}=0$ and $\mu_{-i}=-\mu_{i}$.

Let $\mathscr{G}_{i}$ be the eigenspace of the map ad $\left(H_{\rho}\right)$ corresponding to $\mu_{i}$, for $i=$ $0, \pm 1, \ldots, \pm s$. Note that when $i=0, \mathscr{G}_{i}$ is $\mathscr{G}_{0}$ as defined earlier. Each $\mathscr{G}_{i}$ is a direct sum of $\mathscr{G}_{\beta}$ 's, where $\beta$ is a root satisfying $\beta\left(H_{\rho}\right)=\mu_{i}$. We have the following orthogonal decomposition of $\mathscr{G}$.

$$
\mathscr{G}=\sum_{i=-s}^{s} \mathscr{G}_{i}
$$

Now fix a typical path $w$. We may assume $\theta_{\infty}=H_{\rho} /\left\|H_{\rho}\right\|$. Fix $u \in O(V)$ and recall that $G_{u}$ is defined to be the orbit of $u$ under the action of $G$ on $V$ and it is an $F_{t}$-invariant subbundle of $O(V)$. With the natural identification of $G_{u}$ with $G$, we may identify $T_{u} G_{u}$ with $\mathscr{G}$. The following corollary is a direct consequence of Theorem 3 and (6). 
Corollary 2. Consider the canonical stochastic flow $F_{t}$ restricted to $G_{u}$ for some orthonormal frame $u$ based at $o \in V=G / K$. Let $\theta_{\infty} \in T_{\mathscr{O}} V$ be the limiting direction of the Brownian motion which is the image of $F_{t}(u)$ under the natura! projection: $O(V) \rightarrow V$. With the identification of $T_{o} V$ and $\mathscr{P}$, we may think of $\theta_{\infty}$ as an element of $\mathscr{G} \equiv T_{u} G_{u}$. The linear map $\operatorname{ad}\left(\theta_{\infty}\right): \mathscr{G} \rightarrow \mathscr{G}$ has the following eigenvalues

$$
-\mu_{1}<-\mu_{2}<\cdots<-\mu_{s}<0<\mu_{s}<\cdots<\mu_{2}<\mu_{1} .
$$

Let $\mathscr{G}_{i}^{\prime}$ be the eigenspace corresponding to $\mu_{i}$, for $i=0, \pm 1, \ldots, \pm s$, where $\mu_{0}=0$ and $\mu_{-i}=-\mu_{i}$. Then if $W \in \mathscr{G}_{i}^{\prime}$ with $W \neq 0$,

$$
\lim _{t \rightarrow \infty} \frac{1}{t} \log \left\|D F_{t}(W)\right\|=-\mu_{i} \text {. }
$$

Note that $\mathscr{G}_{i}^{\prime}=\mathscr{G}_{i}$ if $\theta_{\infty} \in \mathscr{A}^{+}$. The following theorem establishes the existence of the so-called stable manifold for the flow $F_{t}$ restricted to $G_{u}$.

Theorem 4. Let $G_{u}^{-}=\left\{e^{X} u ; X \in \sum_{i=1}^{s} \mathscr{G}_{i}^{\prime}\right\}$ and let $d$ be the distance on $G_{u}$ induced by the canonical metric. Then for any $v_{1}, v_{2} \in G_{u}^{-}, d\left(F_{t}\left(v_{1}\right), F_{t}\left(v_{2}\right)\right)$ tends to zero exponentially fast as $t \rightarrow \infty$ in the sense that

$$
\lim _{t \rightarrow \infty} \frac{1}{t} \log d\left(F_{t}\left(v_{1}\right), F_{t}\left(v_{2}\right)\right) \leq-\mu_{s} .
$$

Proof. We may assume $\theta_{\infty} \in \mathscr{A}^{+}$and $\mathscr{G}_{i}^{\prime}=\mathscr{G}_{i}$. It suffices to prove the above for $v_{1}=u$ and $v_{2}=e^{X} u, X \in \mathscr{G}_{i}, i=1,2, \ldots, s$. The distance $d$ on $G_{u}$ is $G$-invariant and it is easy to see that when $X$ is contained in a neighborhood of 0 in $\mathscr{G}, d\left(u, e^{X} u\right) \leq c\|X\|$ for some constant $c>0$. We have

$$
\begin{aligned}
d\left(F_{t}(u), F_{t}\left(e^{X} u\right)\right) & =d\left(g_{t} u, e^{X} g_{t} u\right)=d\left(u, g_{t}^{-1} e^{X} g_{t} u\right) \\
& =d\left(u, e^{\operatorname{Ad}\left(g_{t}^{-1}\right) X} u\right) \leq c\left\|\operatorname{Ad}\left(g_{t}^{-1}\right) X\right\| .
\end{aligned}
$$

It now follows from (36) that if $X$ is a nonzero vector contained in $\mathscr{G}_{i}$ for $i=1,2, \ldots, s$, then

$$
\lim _{t \rightarrow \infty} \frac{1}{t} \log d\left(F_{t}(u), F_{t}\left(e^{X} u\right)\right) \leq-\mu_{i}
$$

Theorem 4 is proved.

For $G=S L(n)$ and $K=S O(n)$, by the root space structure and (13), $s=n-1$ and

$$
\mu_{1}=\frac{n-1}{2 n}, \mu_{2}=\frac{n-2}{2 n}, \ldots, \mu_{n-1}=\frac{1}{2 n} .
$$

Let $g_{t}$ be the horizontal diffusion in $S L(n)$ defined by (7). We have seen that the process $x_{t}=g_{t} g_{t}^{*}$ is the projection of $g_{t}$ into $V$. Let $x_{t}=e^{r_{t} \theta_{t}}$ be the polar decomposition of $x_{t}$, where $r_{t}$ is a positive real-valued process such that $r_{t} / t$ tends to

$$
\left\|H_{\rho}\right\|=\sqrt{\left(n^{2}-1\right) / 24}
$$

the process $\theta_{t}$ is $S$-valued and the limit $\theta_{\infty}=\lim _{t \rightarrow \infty} \theta_{t}$ is a symmetric matrix having eigenvalues (16). The eigenvalues of the linear map $\operatorname{ad}\left(\theta_{\infty}\right): s l(n) \rightarrow$ $s l(n)$ are given by $\mu_{i}, i=0, \pm 1, \ldots, \pm(n-1)$, where $\mu_{0}=0$ and $\mu_{-i}=$ 
$-\mu_{i}$. By Theorem 3, if $W$ in $s l(n)$ is contained in the eigenspace of $\mu_{i}$, then $\left\|g_{t}^{-1} W g_{t}\right\|^{1 / t}$ tends to $e^{-\mu_{i}}$ as $t \rightarrow \infty$, where $\|\cdot\|$ can be any norm on $\operatorname{sl}(n)$.

\section{Proof of Lemma 10}

As before, let $W_{1}, \ldots, W_{n}$ be an orthonormal basis of $\mathscr{G}$ such that each $W_{j}$ is contained in some root space $\mathscr{G}_{\beta}$, let $Z_{1}, \ldots, Z_{h}$ be an orthonormal basis of $\mathscr{K}$ such that each $Z_{i}$ is contained in some $\mathscr{K}_{\alpha}$ for $1 \leq i \leq l$ and $Z_{i} \in \mathscr{M}$ for $i>l$, and let $Y_{1}, \ldots, Y_{l}$ be a basis of $\sum_{\alpha \in \Sigma^{+}} \mathscr{P}_{\alpha}$ such that each $Y_{i}$ is contained in some $\mathscr{P}_{\alpha}$ and $Y_{i}+Z_{i} \in \mathscr{G}_{\alpha}$. Recall that $\beta_{j}$ is the root $\beta$ such that $X_{j} \in \mathscr{G}_{\beta}$. Let $\alpha_{i}$ be the positive root $\alpha$ such that $Y_{i} \in \mathscr{P}_{\alpha}$ and $Z_{i} \in \mathscr{K}_{\alpha}$. We have $-Y_{i}+Z_{i} \in \mathscr{G}_{-\alpha}$,

$$
\left[Z_{i}, X_{j}\right]=\frac{1}{2}\left[Y_{i}+Z_{i}, X_{j}\right]+\frac{1}{2}\left[-Y_{i}+Z_{i}, X_{j}\right] \in \mathscr{G}_{\beta_{j}+\alpha}+\mathscr{G}_{\beta_{j}-\alpha}
$$

and

$$
\operatorname{ad}\left(Z_{i}\right) W_{r}=\sum_{k=1}^{n} C_{i r k} W_{k},
$$

where $C_{i r k}=0$ if $\beta_{k} \neq \beta_{r} \pm \alpha_{i}$.

Fix $\varepsilon>0$. Recall that we have assumed that

$$
x_{t}=\operatorname{Ad}\left(h_{t}\right) A_{t}=\exp \left(\sum_{p=1}^{l} b_{p}\left(x_{t}\right) \operatorname{ad}\left(Z_{p}\right)\right) A_{t},
$$

where $b_{p}\left(x_{t}\right)$ tends to zero and $A_{t} / t$ tends to $H_{\rho}$ as $t \rightarrow \infty$. Let $N$ be the integer in Lemma 9. For $n \geq N$, let $\gamma_{n}$ be the minimal geodesic joining $x_{n}$ and $x_{n+1}$. Since $A_{n}=H_{\rho} n+o(n)$ and the length of $\gamma_{n}$ is at most $n^{\eta}$, where $0<\eta<1$, it follows that the $\mathscr{A}^{+}$-component of $\gamma_{n}$ is also equal to $H_{\rho} n+o(n)$. By (34), we see that along $\gamma_{n}$, when $N$ is sufficiently large,

$$
\left|\sum_{p=1}^{l} a_{i p}(b) d b_{p}\right| \leq C e^{-(1-\varepsilon) \alpha_{i}\left(H_{\rho}\right) n} d s, \quad \text { for } i=1,2, \ldots, l .
$$

Similarly, by Lemma 8 , we obtain, along $\gamma_{n}$,

$$
|d b| \leq C e^{-(1-\varepsilon) \lambda n} d s .
$$

Recall $\lambda=\min _{\alpha \in \Sigma^{+}} \alpha\left(H_{\rho}\right)$. We can introduce a new parameter $u_{n}$ and $\gamma_{n}$ such that $d u_{n}=e^{-(1-\varepsilon) \lambda(n-N)} d s$ We then have

$$
\left|\sum_{p=1}^{l} a_{i p}(b) d b_{p}\right| \leq C e^{-(1-\varepsilon) \alpha_{i}\left(H_{\rho}\right) N} d u_{n}, \quad \text { for } i=1,2, \ldots, h,
$$

where $\alpha_{i}$ is defined to be zero if $i=l+1, \ldots, h$, and

$$
\sum_{n \geq N} \int_{\gamma_{n}}\left|d u_{n}\right| \leq \sum_{n \geq N} e^{-(1-\varepsilon) \lambda(n-N)} n^{\eta} \leq N^{\eta} \sum_{k=0}^{\infty} e^{-(1-\varepsilon) k}(k+1)^{\eta} \leq L N^{\eta},
$$

where $L$ is a positive constant independent of small $\varepsilon>0$. By piecing these parameters $u_{n}$ together we can define a piecewise smooth map $\psi:(0, U] \rightarrow R^{l}$ 
such that $U \leq L N^{\eta}, \psi(U)=b\left(x_{N}\right), \psi(u) \rightarrow 0$ as $u \rightarrow 0$ and

$$
\left|\sum_{p=1}^{l} a_{i p}(\psi) \frac{d}{d u} b_{p}\right| \leq C e^{-(1-\varepsilon) \alpha_{i}\left(H_{\rho}\right) N}, \quad \text { for } i=1,2, \ldots, h,
$$

where $\alpha_{i}$ is $\alpha \in \Sigma^{+}$if $Y_{i} \in \mathscr{P}_{\alpha}$ for $i=1,2, \ldots, l$ and $\alpha_{i}=0$ for $i=$ $l+1, \ldots, h$.

By (30),

$$
\begin{gathered}
\exp \left(-\sum_{j}\left(b_{j}+\Delta b_{j}\right) Z_{j}\right)=\exp \left(-\sum_{i, j} a_{i j} \Delta b_{j} Z_{i}+O\left(|\Delta b|^{2}\right)\right) \exp \left(-\sum_{j} b_{j} Z_{j}\right) \\
\operatorname{Ad}\left(e^{-\sum_{j}\left(b_{j}+\Delta b_{j}\right) Z_{j}}\right)=\left[I-\sum_{i, j} a_{i j} \Delta b_{j} \operatorname{ad}\left(Z_{i}\right)+O\left(|\Delta b|^{2}\right)\right] \operatorname{Ad}\left(e^{-\sum_{j} b_{j} Z_{j}}\right)
\end{gathered}
$$

Recall $f_{j k}$ are defined by (37). We have

$$
\begin{aligned}
\sum_{k=1}^{n} d f_{j k} W_{k} & =d\left[\operatorname{Ad}\left(e^{-\sum_{p} b_{p} Z_{p}}\right) W_{j}\right] \\
& =-\sum_{i=1}^{h} \sum_{p=1}^{l} a_{i p} d b_{p} \operatorname{ad}\left(Z_{i}\right)\left[\operatorname{Ad}\left(e^{-\sum_{p} b_{p} Z_{p}}\right) W_{j}\right] \\
& =-\sum_{i=1}^{h} \sum_{p=1}^{l} \sum_{r=1}^{n} a_{i p} d p_{p} f_{j r} \operatorname{ad}\left(Z_{i}\right) W_{r} \\
& =-\sum_{k=1}^{n} \sum_{i=1}^{h} \sum_{r=1}^{n} C_{i r k} f_{j r}\left(\sum_{p=1}^{l} a_{i p} d b_{p}\right) W_{k}
\end{aligned}
$$

Hence,

$$
d f_{j k}=-\sum_{i=1}^{h} \sum_{r=1}^{n} C_{i r k} f_{j r}\left(\sum_{p=1}^{l} a_{i p} d b_{p}\right)
$$

Let

and

$$
g_{j k}=f_{j k} e^{(1-\varepsilon)\left|\beta_{j}-\beta_{k}\right|\left(H_{\rho}\right) N}
$$

$$
d v_{i}=e^{(1-\varepsilon) \alpha_{i}\left(H_{\rho}\right) N} \sum_{p=1}^{l} a_{i p} d b_{p} .
$$

Since $C_{i r k}=0$ if $\beta_{k} \neq \beta_{r} \pm \alpha_{i}$ and $\left(\left|\beta_{j}-\beta_{r}\right|+\alpha_{i}\right)\left(H_{\rho}\right) \geq\left|\beta_{j}-\beta_{k}\right|\left(H_{\rho}\right)$ if $\beta_{k}=\beta_{r} \pm \alpha_{i}$, it follows that

$$
d g_{j k} \sum_{i=1}^{h} \sum_{r=1}^{n} \widetilde{C}_{i r k} g_{j r} d v_{i}
$$

where $\left|\widetilde{C}_{i r k}\right| \leq\left|C_{i r k}\right|$. With the map $\psi$ defined above, we can regard $b_{i}, f_{j k} \circ \psi$ and $g_{j k} \circ \psi$ as piecewise smooth functions of $u$ and by (42), we have $\left|d v_{i} / d u\right| \leq$ $C$. It follows that $g_{j k} \circ \psi$ satisfy a system of linear homogeneous differential 
equations

$$
\frac{d}{d u} g_{j k} \circ \psi=\sum_{r} C_{r k}(u) g_{j r} \circ \psi,
$$

with bounded coefficients $C_{r k}(u)$.

Now fix $j$. Let $\left|g_{j}\right|$ be the square root of $\sum_{k}\left|g_{j k} \circ \psi\right|^{2}$. Then there exists a constant $\Lambda>0$ such that $\left|g_{j}\right|(U) \leq\left|g_{j}\right|(0) e^{\Lambda U} \leq\left|g_{j}\right|(0) e^{\Lambda L N^{\eta}}$. Since $f_{j k}(0)=$ $\delta_{j k}$, we see that $\left|g_{j}\right|(\mathrm{G})=\left|f_{j j}(0)\right|=1$. Hence,

$$
\left|f_{j k} \circ \psi(U)\right|=\left|g_{j k} \circ \psi(U)\right| e^{-(1-\varepsilon)\left|\beta_{j}-\beta_{k}\right|\left(H_{\rho}\right) N} \leq e^{\Lambda L N^{\eta}} e^{-(1-\varepsilon)\left|\beta_{j}-\beta_{k}\right|\left(H_{\rho}\right) N} .
$$

If $\beta_{j} \neq \beta_{k},\left|\beta_{j}-\beta_{k}\right|\left(H_{\rho}\right)>0$. Since $0<\eta<1, \Lambda L N^{\eta} \leq \varepsilon\left|\beta_{j}-\beta_{k}\right|\left(H_{\rho}\right) N$ when $N$ is sufficiently large. It follows that

$$
\left|f_{j k}\left(b\left(x_{N}\right)\right)\right|=\left|f_{j k} \circ \psi(U)\right| \leq e^{-(1-2 \varepsilon)\left|\beta_{j}-\beta_{k}\right|\left(H_{\rho}\right) N} .
$$

It is easy to see that in the previous discussion the integer $N$ can be replaced by any large real number $t$. We obtain (39) by replacing $2 \varepsilon$ by $\varepsilon$. Lemma 10 is proved.

\section{ACKNOWLEDGMENT}

I wish to thank M. Pinsky for inviting me to visit Northwestern University in the summer of 1990 during which time the paper was written. I am also very grateful to the referee of this paper for his careful reading of the paper and for his valuable comments which have led to important changes in the form of the paper.

\section{REFERENCES}

1. P. H. Baxendale, Asymptotic behaviour of stochastic flows of diffeomorphisms: two case studies, Probab. Theory Related Fields 73 (1986), 51-85.

2. A. P. Carverhill and K. D. Elworthy, Lyapunov exponents for a stochastic analogue of the geodesic flow, Trans. Amer. Math. Soc. 295 (1986), 85-105.

3. S. Helgason, Differential geometry, Lie groups, and symmetric spaces, Academic Press, 1978.

4. _. Groups and geometric analysis, Academic Press, 1984.

5. M. Liao, Stochastic flows on the boundaries of Lie groups, Stochastics Stochastics Rep. 39 (1992), 213-237.

6. M. P. Malliavin and P. Malliavin, Factorisations et lois limites de la diffusion horizontale au-dessus d'un espace Riemannien symmetrique, Lecture Notes in Math., vol. 404, SpringerVerlag, 1974, pp. 164-217.

7. J. R. Norris, L. C. G. Rogers, and D. Williams, Brownian motion of ellipsoids, Trans. Amer. Math. Soc. 296 (1986), 757-765.

8. A. Orihara, On random ellipsoid, J. Fac. Sci. Univ. Tyoko Sect. IA Math. 17 (1970), 73-85.

9. M. Pinsky, Stochastic Riemannian geometry, Probabilistic Analysis and Related Topics, vol. 1 (A. T. Bharucha Reid, ed.), Academic Press, 1978.

10. M. J. Prat, Etude asymptotique et convergence angulaire du mouvement brownien sur une variété à courbure négative, C. R. Acad. Sci. Paris Sér. A 280 (1975), 1539-1542.

11. J. C. Taylor, The Iwasawa decomposition and the limiting behavior of Brownian motion on a symmetric space of non-compact type, Contemp. Math., vol. 73, Amer. Math. Soc., Providence, R.I., 1988, pp. 303-332.

12. __ Brownian motion on a symmetric space of non-compact type: asymptotic behavior in polar coordinates, Canad. J. Math. 43 (1991), 1065-1085. 